Supplementary information for

\title{
Rhodium-Catalyzed Regioselective Hydroformylation of Alkynes to $\alpha, \beta$-Unsaturated Aldehydes using Formic Acid
}

\author{
Chao Fan ${ }^{1, \uparrow}$, Jing Hou ${ }^{1, \uparrow}$, Yu-Jia Chen ${ }^{1}$, Kui-Ling Ding ${ }^{2, *}$ and Qi-Lin Zhou ${ }^{1, *}$ \\ ${ }^{1}$ State Key Laboratory and Institute of Elemento-Organic Chemistry, College of Chemistry, \\ Nankai University, Tianjin 300071, P. R. China. \\ ${ }^{2}$ State Key Laboratory of Organometallic Chemistry, Shanghai Institute of Organic \\ Chemistry, Chinese Academy of Sciences, 345Lingling Road, Shanghai 200032, P.R. China \\ ${ }^{\dagger}$ Chao Fan and Jing Hou contributed equally to this work. \\ Correspondence to: qlzhou@nankai.edu.cn
}

\section{Table of Contents}

1. General information .........................................................................

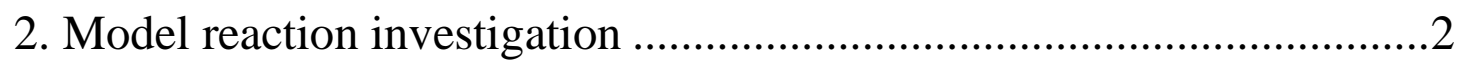

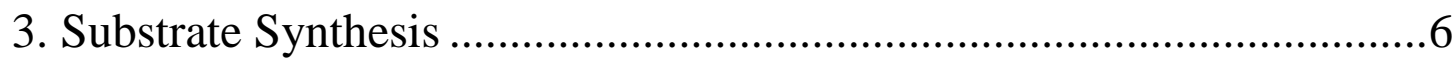

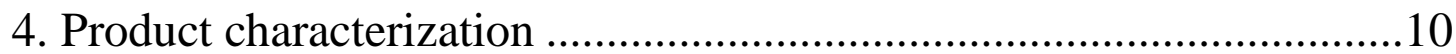

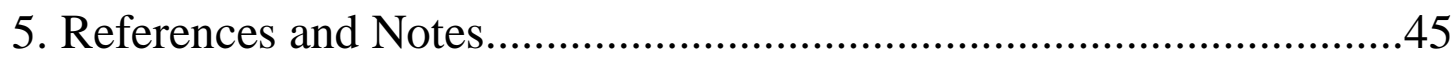




\section{General information}

Unless mentioned otherwise, all manipulations were performed in an argon-filled glove box MBRAUN LABstar or using standard Schlenk techniques. NMR spectra were recorded on a Bruker AV 400 spectrometer at $400 \mathrm{MHz}\left({ }^{1} \mathrm{H} \mathrm{NMR}\right), 101 \mathrm{MHz}\left({ }^{13} \mathrm{C}\right.$ NMR). Chemical shifts were reported in ppm related to internal TMS for ${ }^{1} \mathrm{H}$ NMR data, deuterated solvent for ${ }^{13} \mathrm{C}$ NMR data, respectively. Data are presented in the following space: chemical shift, multiplicity, coupling constant in hertz $(\mathrm{Hz})$, and signal area integration in natural numbers. High-resolution mass spectra were recorded on an IonSpec FT-ICR mass spectrometer with ESI or MALDI resource. GC analysis was performed using Agilent Technologies 7890A GC System or Fuli GC 9790 II system. Capillary column HP-INNOWAX, HP-5, TDX-01 was used for GC quantitative analysis.

Diethyl ether, tetrahydrofuran and toluene that were used for substrate synthesis were treated with MBRAUN MB-SPS-5 solvent purification system. For solvents that were used for hydroformylation were dried by distillation with sodium or $\mathrm{CaH}_{2}$ under argon. They were degassed before use. $\mathrm{Rh}(\mathrm{CO})_{2}(\mathrm{acac})$ was purchased from Strem Chemicals Co. It can be purified by sublimation if it is not pure enough. Acetic anhydride was distilled. Formic acid was purchased from Aladdin Bio-Chem Technology Co. and used without further purification. Different sources of formic acid could have significant effect on the reaction results. Using boric anhydride as drying reagent and distilling the formic acid at $25{ }^{\circ} \mathrm{C} / 40 \mathrm{mmHg}$ can guarantee the reaction's reproducibility. Other commercially available reagents were purchased from Adamas, Acros, Alfa, Heowns and TCI Chemical Companies. Unless mentioned otherwise, further purification of reagents is not needed. 


\section{Model reaction investigation}

\section{Standard condition A (Auto-clave)}

An oven-dried $15 \mathrm{~mL}$ autoclave with a glass vessel was charged with a stir bar, Xantphos (11.6 mg $0.02 \mathrm{mmol}), \mathrm{Rh}(\mathrm{acac})(\mathrm{CO})_{2}(2.6 \mathrm{mg}, 0.01 \mathrm{mmol})$ and alkyne substrate $(0.5 \mathrm{mmol})$. The autoclave was evacuated and refilled with argon. Then toluene $(3 \mathrm{~mL})$ was injected. The mixture was stirred for $1 \mathrm{~min}$. Then $\mathrm{HCOOH}(4.5$ $\mathrm{mmol}, 207 \mathrm{mg})$ and $\mathrm{Ac}_{2} \mathrm{O}(3 \mathrm{mmol}, 306 \mathrm{mg})$ were added to the mixture quickly. The mixture was stirred for $1 \mathrm{~min}$ and heated at $100{ }^{\circ} \mathrm{C}$ in an oil bath for $12 \mathrm{~h}$. Pressure readings during the reaction ranged from 0.7 to $1.0 \mathrm{MPa}$. After cooling to room temperature, $\mathrm{CO}$ gas was carefully released in a well-ventilated fume hood. Solvents and acid were removed under reduced pressure, and the residue was purified by column chromatography on silica gel (PE/EA, v/v $=50: 1)$.

Note: If alkyne is liquid, it should be added after toluene was injected.

\section{Standard condition B (Sealed tube)}

In an argon-filled glove box, an oven-dried $15 \mathrm{~mL}$ sealed tube was charged with a stir bar, Xantphos (11.6 mg $0.02 \mathrm{mmol}), \mathrm{Rh}(\mathrm{acac})(\mathrm{CO})_{2}(2.6 \mathrm{mg}, 0.01 \mathrm{mmol})$ and alkyne substrate $(0.5 \mathrm{mmol})$. The toluene $(3 \mathrm{~mL})$ was injected into the tube. The mixture was stirred for $1 \mathrm{~min}$. $\mathrm{HCOOH}\left(4.5 \mathrm{mmol}, 207 \mathrm{mg}\right.$ ) and $\mathrm{Ac}_{2} \mathrm{O}$ (3 mmol, $306 \mathrm{mg}$ ) were added to the mixture quickly. The mixture was stirred for 1 min and wrapped with tin foil to avoid light exposure. The mixture was heated at $100{ }^{\circ} \mathrm{C}$ in an oil bath for $12 \mathrm{~h}$. After cooling to room temperature, $\mathrm{CO}$ gas was carefully released in a well-ventilated fume hood. Solvents and acid were removed under reduced pressure, and the residue was purified by column chromatography on silica gel $(\mathrm{PE} / \mathrm{EA}, \mathrm{v} / \mathrm{v}=50: 1)$.

Standard condition $\mathbf{C}$ (gram scale experiment)

An oven-dried $150 \mathrm{~mL}$ autoclave with a glass vessel was charged with a stir bar, Xantphos (144.5 mg $0.25 \mathrm{mmol}), \mathrm{Rh}(\mathrm{acac})(\mathrm{CO})_{2}(32.2 \mathrm{mg}, 0.125 \mathrm{mmol})$ and alkyne substrate $(6.25 \mathrm{mmol})$. The autoclave was evacuated and refilled with argon. Then toluene $(35 \mathrm{~mL})$ was injected. The mixture was stirred for $3 \mathrm{~min}$. HCOOH $(56 \mathrm{mmol}$, $2.6 \mathrm{~g})$ and $\mathrm{Ac}_{2} \mathrm{O}(37.5 \mathrm{mmol}, 3.8 \mathrm{~g})$ were added to the mixture quickly. The mixture was stirred for $3 \mathrm{~min}$ and heated at $100{ }^{\circ} \mathrm{C}$ in an oil bath for $12 \mathrm{~h}$. Pressure readings during the reaction ranged from 0.8 to $1.1 \mathrm{MPa}$. After cooling to room temperature, $\mathrm{CO}$ gas was carefully released in a well-ventilated fume hood. Solvents and acid were removed under reduced pressure, and the residue was purified by column chromatography on silica gel (PE/EA, v/v = 50:1). $1.32 \mathrm{~g}, 87 \%$ of $\mathbf{2 a}$ was obtained. 
Table S1. Ligand screening for hydroformylation

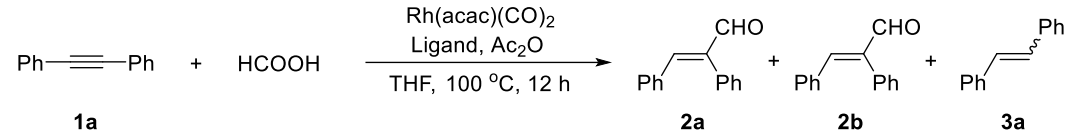<smiles>Pc1cccc(P)c1P</smiles><smiles>c1ccc(-c2ccccc2-c2ccccc2-c2ccccc2)cc1</smiles>

L2

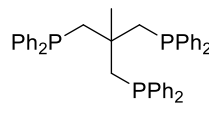

L3

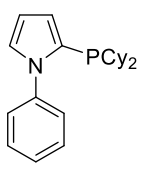

L4

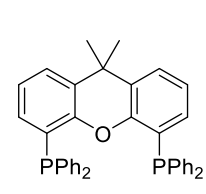

L5

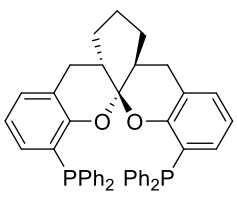

L6
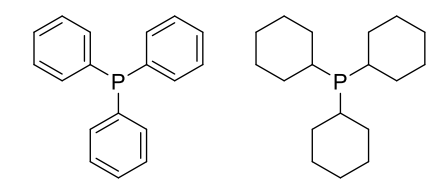

$\mathrm{Ph}_{2} \mathrm{P} \sim \mathrm{PPh}_{2} \mathrm{Ph}_{2} \mathrm{P} \sim \mathrm{PPh}_{2}$
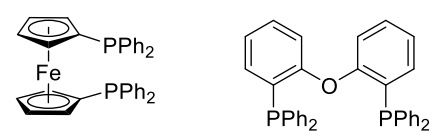

L7

L8

L9

L10

L11

L12

\begin{tabular}{cccc}
\hline Entry $^{\mathrm{a}}$ & Ligand & Yield [\%] 2a/2b & Yield [\%] 3a \\
\hline 1 & L1 & -- & 100 \\
2 & $\mathbf{L 2}$ & -- & 100 \\
3 & $\mathbf{L 3}$ & -- & 100 \\
4 & $\mathbf{L 4}$ & -- & 100 \\
5 & $\mathbf{L 5}$ & $25 / 2$ & 70 \\
6 & $\mathbf{L 6}$ & $11 /$ trace & 85 \\
7 & $\mathbf{L 7}$ & -- & 100 \\
8 & $\mathbf{L 8}$ & -- & 80 \\
9 & $\mathbf{L 9}$ & $0 / 23$ & 77 \\
10 & $\mathbf{L 1 0}$ & $2 / 2$ & 95 \\
11 & $\mathbf{L 1 1}$ & $1 / 1$ & 94 \\
12 & $\mathbf{L 1 2}$ & -- & 100 \\
\hline
\end{tabular}

${ }^{a}$ Reaction condition: 1a $(0.5 \mathrm{mmol}), \mathrm{HCOOH}(1.5 \mathrm{mmol}), \mathrm{Ac}_{2} \mathrm{O}(0.75 \mathrm{mmol}), \mathrm{Rh}(\mathrm{acac})(\mathrm{CO})_{2}$ (0.005 mmol), ligand $(0.01 \mathrm{mmol}), \operatorname{THF}(3 \mathrm{~mL}), 100{ }^{\circ} \mathrm{C}, 12 \mathrm{~h}$. Yields were determined by ${ }^{1} \mathrm{H}$ NMR analysis using 1,3,5-trimethoxybenzene as internal standard. 
Table S2. Condition screening for hydroformylation

\begin{tabular}{|c|c|c|c|c|c|}
\hline & $\overline{\overline{1 a}} \mathrm{Ph}$ & $+\mathrm{HCOOH}$ & $\begin{array}{c}\mathrm{Rh}(\mathrm{acac})(\mathrm{CO})_{2} \\
\text { Xantphos, } \mathrm{Ac}_{2} \mathrm{O} \\
\text { Solvent, } 100{ }^{\circ} \mathrm{C}, 12 \mathrm{~h}\end{array}$ & $\mathrm{Ph}_{2 \mathrm{a}}^{\mathrm{CHO}}{ }_{\mathrm{Ph}}^{\mathrm{CHO}}$ & $={ }_{\mathrm{Ph}}^{\mathrm{CHO}}+{ }_{\mathrm{Ph}} \overbrace{\mathbf{3 a}}^{{ }_{5}^{5 h}}$ \\
\hline \multirow[t]{2}{*}{ Entry $^{\mathrm{a}}$} & $\mathrm{L} / \mathrm{Rh}$ & $\begin{array}{c}\mathrm{HCOOH} \\
(\mathrm{mmol})\end{array}$ & $\begin{array}{c}\mathrm{Ac}_{2} \mathrm{O} \\
(\mathrm{mmol})\end{array}$ & Solvents & Yield $[\%]^{[b]} \mathbf{2 a} / \mathbf{2 b}$ \\
\hline & 1 & 1.5 & 0.75 & THF & -- \\
\hline $2^{\mathrm{c}}$ & 1 & 1.5 & 0.75 & THF & $23 / 3$ \\
\hline 3 & 1 & 1.5 & 0.75 & Toluene & $31 / 0$ \\
\hline 4 & 1 & 1.5 & 0.75 & DMF & $21 / 0$ \\
\hline 5 & 1 & 1.5 & 0.75 & DME & $27 / 0$ \\
\hline 6 & 1 & 3 & 1.5 & Toluene & $33 / 0$ \\
\hline 7 & 2 & 3 & 1.5 & Toluene & $55 / 2$ \\
\hline 8 & 3 & 3 & 1.5 & Toluene & $55 / 2$ \\
\hline 9 & 2 & 3 & 0 & Toluene & -- \\
\hline 10 & 2 & 3 & 1 & Toluene & $50 / 0$ \\
\hline 11 & 2 & 3 & 2 & Toluene & $56 / 0$ \\
\hline 12 & 2 & 3 & 3 & Toluene & $54 / 0$ \\
\hline 13 & 2 & 4.5 & 1.5 & Toluene & $43 / 0$ \\
\hline 14 & 2 & 4.5 & 2.25 & Toluene & $58 / 0$ \\
\hline 15 & 2 & 4.5 & 3 & Toluene & $73 / 0$ \\
\hline 16 & 2 & 4.5 & 4.5 & Toluene & $70 / 0$ \\
\hline 17 & 3 & 4.5 & 3 & Toluene & $73 / 0$ \\
\hline 18 & 2 & 6 & 4 & Toluene & $22 / 1$ \\
\hline $19^{d}$ & 2 & 4.5 & 3 & Toluene & $40 / 1$ \\
\hline $20^{\mathrm{e}}$ & 2 & 4.5 & 3 & Toluene & $20 / 1$ \\
\hline $21^{\mathrm{f}}$ & 2 & 4.5 & 3 & Toluene & $85 / 2$ \\
\hline $22^{\mathrm{f}, \mathrm{g}}$ & 2 & 4.5 & 3 & Toluene & $33 / 1$ \\
\hline $23^{\mathrm{f}, \mathrm{h}}$ & 2 & 4.5 & 3 & Toluene & $58 / 1$ \\
\hline
\end{tabular}

a Reaction condition: 1a $(0.5 \mathrm{mmol}), \mathrm{HCOOH}(1.5-4.5 \mathrm{mmol}), \mathrm{Ac}_{2} \mathrm{O}(0.75-4.5 \mathrm{mmol})$, $\mathrm{Rh}(\mathrm{acac})(\mathrm{CO})_{2}(0.005 \mathrm{mmol})$, Xantphos $(0.005-0.015 \mathrm{mmol})$, solvent $(3 \mathrm{~mL}), 100{ }^{\circ} \mathrm{C}, 12 \mathrm{~h}$. Yields were determined by ${ }^{1} \mathrm{H}$ NMR analysis using 1,3,5-trimethoxybenzene as internal standard. ${ }^{\mathrm{b}}$ $\left[\mathrm{Rh}(\mathrm{CO})_{2} \mathrm{Cl}\right]_{2}$ was used instead. ${ }^{\mathrm{c}} \mathrm{Rh}(\mathrm{acac})\left(\mathrm{C}_{2} \mathrm{H}_{4}\right)_{2}$ was used instead. ${ }^{\mathrm{d}} \mathrm{Piv}_{2} \mathrm{O}$ was used instead. ${ }^{\mathrm{e}}$ $\mathrm{Bz}_{2} \mathrm{O}$ was used instead. ${ }^{\mathrm{f}} \mathrm{Rh}(\mathrm{acac})(\mathrm{CO})_{2}(0.01 \mathrm{mmol})$, Xantphos $(0.02 \mathrm{mmol}) .{ }^{\mathrm{g}} 80^{\circ} \mathrm{C} .{ }^{\mathrm{h}} 120{ }^{\circ} \mathrm{C}$. 
Table S3. Reagent influence on hydroformylation

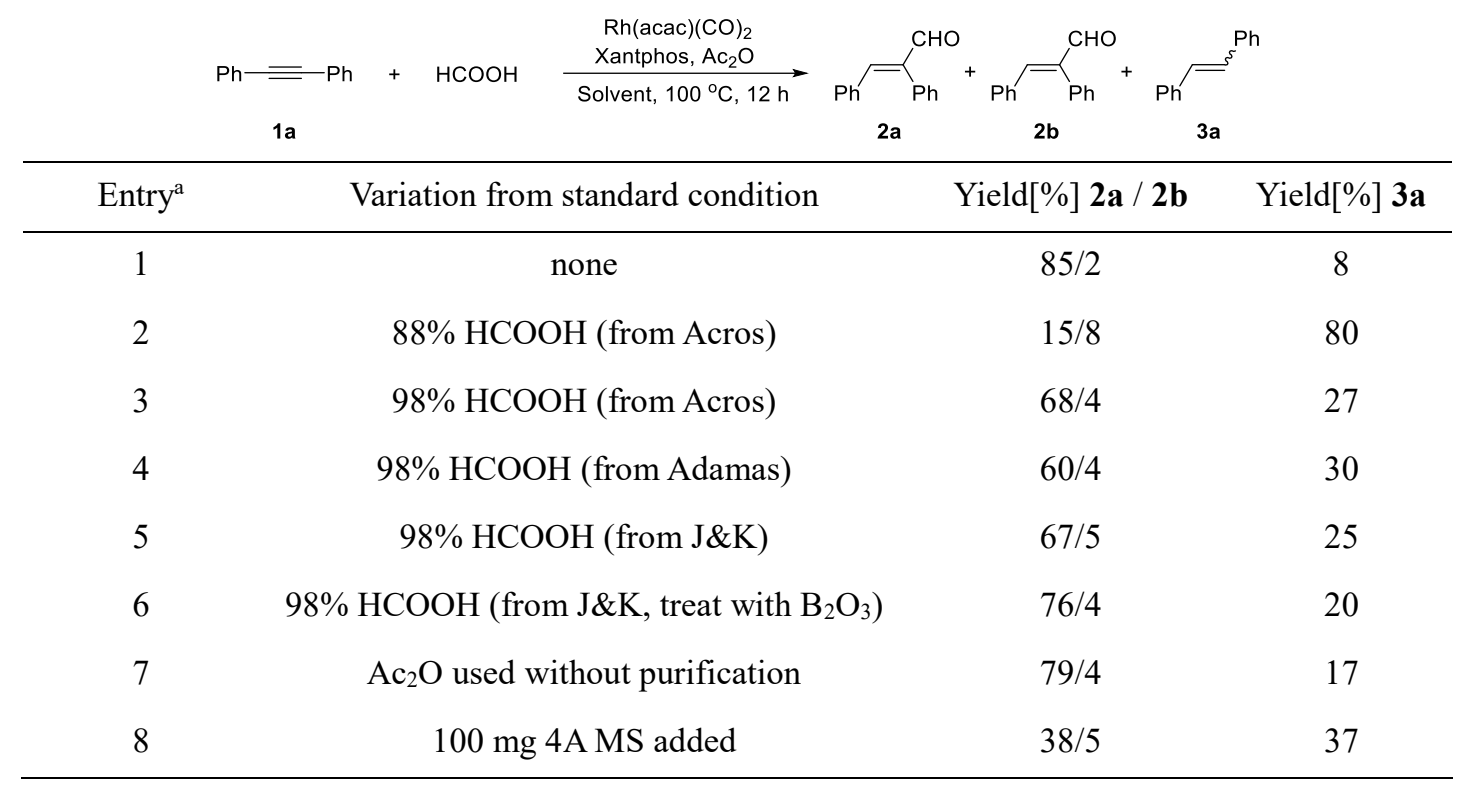

${ }^{a}$ Standard condition: 1a $(0.5 \mathrm{mmol}), \mathrm{HCOOH}\left(4.5 \mathrm{mmol}\right.$, from aladdin), $\mathrm{Ac}_{2} \mathrm{O}(3.0 \mathrm{mmol})$, $\mathrm{Rh}(\mathrm{acac})(\mathrm{CO})_{2}(0.01 \mathrm{mmol})$, Xantphos $(0.02 \mathrm{mmol})$, Toluene $(3 \mathrm{~mL}), 100{ }^{\circ} \mathrm{C}, 12 \mathrm{~h}$. Yields were determined by ${ }^{1} \mathrm{H}$ NMR analysis using 1,3,5-trimethoxybenzene as internal standard.

\section{Notes on reproducibility}

Impurities in starting materials significantly affect the yield and selectivity of the reaction. Alkynes and Xantphos was used without purification. If they were stored for a long time, water should be removed under high vacuum. $\mathrm{Rh}(\mathrm{acac})(\mathrm{CO})_{2}$ was purchased from Strem Chemicals Co. and used without purification. This dichroic compound is green in crystal form and red in powder form. If black impurities were observed, it should be purified by sublimation. ${ }^{1} \mathrm{Ac}_{2} \mathrm{O}$ was purified by distillation at $140{ }^{\circ} \mathrm{C} / 760 \mathrm{mmHg}$. Toluene was distilled from sodium and degassed before use. Different sources of formic acid might contain different impurities owning to different purification methods used. By treatment with freshly prepared $\mathrm{B}_{2} \mathrm{O}_{3}$ for $12 \mathrm{~h}$, water in formic acid can be effectively removed. Further purification of formic acid can be achieved by distillation at $25^{\circ} \mathrm{C} / 40 \mathrm{mmHg}{ }^{2}$ 


\section{Substrate Synthesis}

1a, 1p, 1q, 1r, 1s, 1u, 1v, 1w, 1x and 1z were purchased and used without further purification. Other alkynes were synthesized according to literatures.

\section{Synthesis of symmetrical aryl alkynes (1b-1m) ${ }^{3}$}

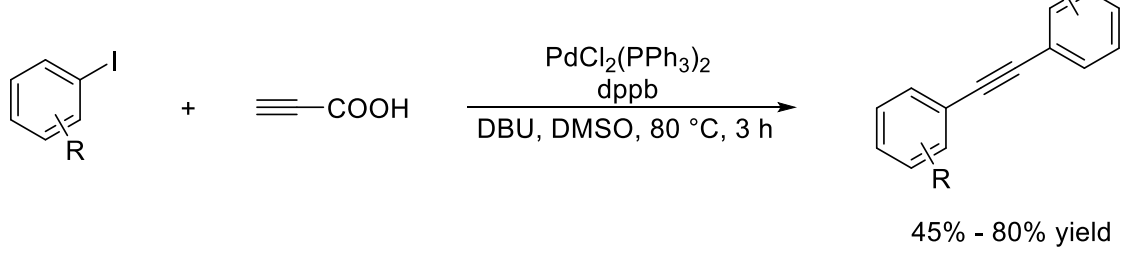

$\mathrm{PdCl}_{2}(\mathrm{PPh} 3)_{2}$ (105 mg, $0.15 \mathrm{mmol}$ ), 1,4-bis(diphenylphosphino)butane (128 mg, $0.30 \mathrm{mmol})$, aryl halides $(6.00 \mathrm{mmol})$, and propiolic acid $(212 \mathrm{mg}, 3.0 \mathrm{mmol})$ were combined with DBU (913 mg, $6.0 \mathrm{mmol})$ in a small round-bottomed flask. DMSO (15.0 $\mathrm{mL}$ ) was added, and the flask was sealed with a septum. The resulting mixture was placed in an oil bath at $80{ }^{\circ} \mathrm{C}$ for $3 \mathrm{~h}$. The reaction was poured into $25 \mathrm{~mL}$ of saturated aqueous ammonium chloride and extracted with $\mathrm{Et}_{2} \mathrm{O}(4 \times 20 \mathrm{~mL})$. The combined ether extracts were washed with brine $(90 \mathrm{~mL})$, dried over $\mathrm{MgSO}_{4}$, and filtered. After removal of solvent under vacuum, the crud product was purified by flash chromatography on silica gel column eluting with petroleum ether/ethyl acetate $(\mathrm{v} / \mathrm{v}=$ 97:3).

Usually, alkyne products are white or pale-yellow solid. Recrystallization from hexane and ethyl acetate can effectively remove the impurities.

Synthesis of alkyne $1 n^{4}$
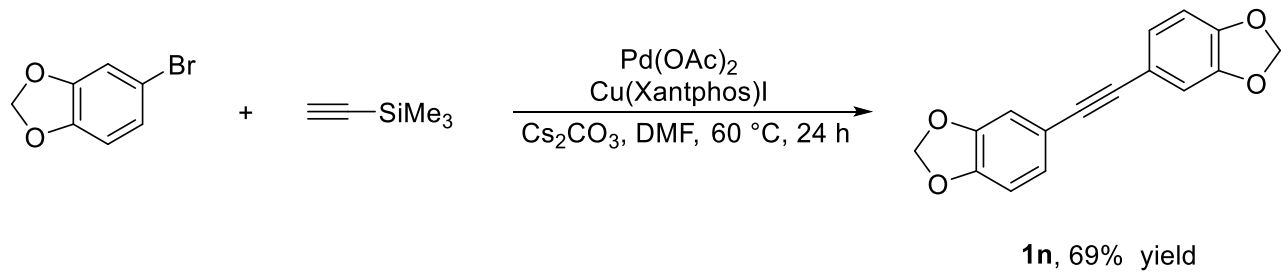

The mixture of piperonyl bromide $(1 \mathrm{~g}, 5 \mathrm{mmol})$ and trimethylsilylethynylene (300 $\mathrm{mg}, 3 \mathrm{mmol}), \mathrm{Pd}(\mathrm{OAc})_{2}(22.4 \mathrm{mg}, 0.1 \mathrm{mmol}), \mathrm{Cu}$ (Xantphos) $\mathrm{I}^{5}$ (76.8 mg, $\left.0.1 \mathrm{mmol}\right)$ and $\mathrm{Cs}_{2} \mathrm{CO}_{3}(3.25 \mathrm{~g}, 10 \mathrm{mmol})$ in anhydrous DMF $(15 \mathrm{~mL})$ was heated at $60{ }^{\circ} \mathrm{C}$ for 24 $\mathrm{h}$ under argon atmosphere. After the reactions were completed, DMF was removed under reduced pressure. The mixture was extracted with ethyl acetate $(3 \times 15 \mathrm{~mL})$ three times, and the combined organic layers were dried over anhydrous $\mathrm{Na}_{2} \mathrm{SO}_{4}$ and filtered. After removal of the solvent, the residue was purified by flash column chromatography on silica gel eluting with petroleum ether to afford the target product in $69 \%$ yield (recrystallized) as an off-white solid. Mp 183-185 ${ }^{\circ} \mathrm{C} .{ }^{1} \mathrm{H}$ NMR $\left(400 \mathrm{MHz}, \mathrm{CDCl}_{3}\right) \delta$ $7.03(\mathrm{dd}, J=8.1,1.5 \mathrm{~Hz}, 2 \mathrm{H}), 6.95(\mathrm{~s}, 2 \mathrm{H}), 6.78(\mathrm{~d}, J=8.0 \mathrm{~Hz}, 2 \mathrm{H}), 5.98(\mathrm{~s}, 4 \mathrm{H}) .{ }^{13} \mathrm{C}$ NMR (101 MHz, $\left.\mathrm{CDCl}_{3}\right) \delta$ 147.76, 147.43, 126.09, 116.64, 111.46, 108.48, 101.29, 87.67. 


\section{Synthesis of alkyne $10^{6}$}<smiles>C#CC(=O)O</smiles>

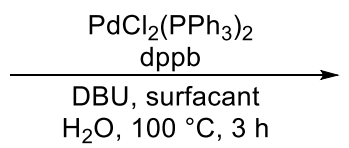<smiles>C(#Cc1ccoc1)c1ccoc1</smiles>

10, $74 \%$ yield

In a $100 \mathrm{~mL}$ Sclenk tube with a cold condenser, $\mathrm{PdCl}_{2}\left(\mathrm{PPh}_{3}\right)_{2}(104 \mathrm{mg}, 0.15 \mathrm{mmol})$, 1,4-bis(diphenylphosphino)butane (128 mg, $0.3 \mathrm{mmol}$ ), octadecyltrimethyl-ammonium chloride $(40 \mathrm{mg})$, furan bromide $(876 \mathrm{mg}, 6 \mathrm{mmol})$ and DBU $(2.28 \mathrm{~g}, 15 \mathrm{mmol})$ were added. The mixture was added distilled $\mathrm{H}_{2} \mathrm{O}(16 \mathrm{~mL})$ and propiolic acid $(210 \mathrm{mg}, 3$ mmol) and stirred under aerobic conditions. The flask was sealed with a septum and stirred at $100{ }^{\circ} \mathrm{C}$ for $3 \mathrm{~h}$. After cooling to room temperature, the reaction mixture was diluted with ethyl acetate $(4 \times 30 \mathrm{~mL})$, washed with brine $(80 \mathrm{~mL})$, dried over $\mathrm{MgSO}_{4}$, and filtered. The solvent was removed under vacuum, and the resulting crude product was purified by flash chromatography on silica gel to give $10(259 \mathrm{mg}, 74 \%$ yield, yellow solid). Product decomposes at room temperature and should be stored at $-20{ }^{\circ} \mathrm{C}$. ${ }^{1} \mathrm{H}$ NMR $\left(400 \mathrm{MHz}, \mathrm{CDCl}_{3}\right) \delta 7.67-7.64(\mathrm{~m}, 2 \mathrm{H}), 7.39(\mathrm{t}, J=1.7 \mathrm{~Hz}, 2 \mathrm{H}), 6.50-6.48$ $(\mathrm{m}, 2 \mathrm{H}) .{ }^{13} \mathrm{C} \mathrm{NMR}\left(101 \mathrm{MHz}, \mathrm{CDCl}_{3}\right) \delta 145.42,142.86,112.44,107.56,82.05$.

\section{Synthesis of alkyne $1 t^{7}$}<smiles>O=C1CCCCCCCCCCC1</smiles>

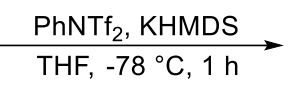<smiles>CCCCCCCCC/C=C(\CCCC)OC</smiles>

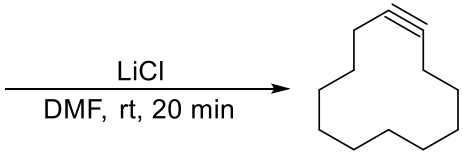

$1 \mathrm{t}$

Step 1. To a stirred solution of cyclodocecanone $(2.0 \mathrm{~g}, 11.0 \mathrm{mmol})$ and $\mathrm{PhNTf}_{2}$ $(4.3 \mathrm{~g}, 12.0 \mathrm{mmol})$ in THF $(40 \mathrm{~mL})$ was added KHMDS $(13.0 \mathrm{~mL}, 1 \mathrm{M}, 13.0 \mathrm{mmol})$ at $-78{ }^{\circ} \mathrm{C}$. After stirring at this temperature for 1 hour, the reaction was quenched with saturated aqueous $\mathrm{NH}_{4} \mathrm{Cl}$ and extracted with ethyl acetate. The enol triflate was purified by column chromatography (hexanes/EA $=200 / 1$ ). Trace impurities can be removed in the next step.

Step 2. The enol triflate $(1.57 \mathrm{~g}, 5.0 \mathrm{mmol})$ was dissolved in $10 \mathrm{~mL}$ of DMF and treated with $\mathrm{LiCl}(848 \mathrm{mg}, 20.0 \mathrm{mmol})$ for 20 minutes. Then the reaction mixture was directly subjected to column chromatography eluting with petroleum ether to give $\mathbf{1 t}$ as pale-yellow liquid (590 mg, 71\%). ${ }^{1} \mathrm{H}$ NMR (400 MHz, $\left.\mathrm{CDCl}_{3}\right) \delta 2.25-2.15(\mathrm{~m}, 4 \mathrm{H})$, $1.61-1.50(\mathrm{~m}, 8 \mathrm{H}), 1.49-1.39(\mathrm{~m}, 8 \mathrm{H}) .{ }^{13} \mathrm{C} \mathrm{NMR}\left(101 \mathrm{MHz}, \mathrm{CDCl}_{3}\right) \delta 81.58,25.67$, $25.57,24.89,24.57,18.49$.

\section{Synthesis of $1 z^{8}$}<smiles>OCC#Cc1ccccc1</smiles>
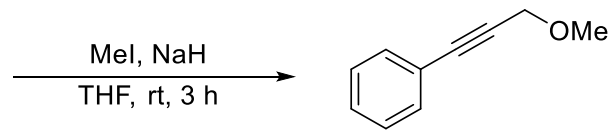

The 3-phenylprop-2-yn-1-ol (1.32 g, $10 \mathrm{mmol})$ was dissolved in $20 \mathrm{~mL}$ of THF and treated with $\mathrm{NaH}$ (480 mg, $20.0 \mathrm{mmol}$ ) (added in small portions). After no more 
hydrogen evolved, MeI (2.82 mg, $20.0 \mathrm{mmol})$ was added dropwise through a syringe. The reaction mixture was stirred at room temperature for $3 \mathrm{~h}$. The reaction was quenched with saturated aqueous $\mathrm{NH}_{4} \mathrm{Cl}$ and extracted with ethyl acetate. The solvent was removed under vacuum and the residue was re-dissolved in petroleum ether. The solution was filtrated through a pad of silica gel. Pure product $\mathbf{1 z}(1.38 \mathrm{~g}, 95 \%)$ was obtained as pale-yellow liquid by distillation at $120{ }^{\circ} \mathrm{C} / 8 \mathrm{mmHg} .{ }^{1} \mathrm{H} \mathrm{NMR}(400 \mathrm{MHz}$, $\left.\mathrm{CDCl}_{3}\right) 7.41-7.35(\mathrm{~m}, 2 \mathrm{H}), 7.26-7.19(\mathrm{~m} 2 \mathrm{H}), 4.25(\mathrm{~s}, 2 \mathrm{H}), 3.38(\mathrm{~s}, 3 \mathrm{H}) .{ }^{13} \mathrm{C} \mathrm{NMR}$ $\left(101 \mathrm{MHz}, \mathrm{CDCl}_{3}\right) \delta 131.75,128.43,128.28,122.65,86.38,84.90,60.43,57.68$.

\section{Synthesis of acetic formic anhydride9}

To a solution of sodium formate $(10 \mathrm{~g}, 0.15 \mathrm{~mol}, 1.2 \mathrm{eq})$ in dry $\mathrm{Et}_{2} \mathrm{O}(32 \mathrm{~mL})$, acetyl chloride (9.8 g, $0.124 \mathrm{~mol}, 1 \mathrm{eq})$ was added dropwise, keeping the temperature below $30{ }^{\circ} \mathrm{C}$. The mixture was stirred overnight under nitrogen at $25{ }^{\circ} \mathrm{C}$. The solids were separated by filtration and the solution was concentrated under reduced pressure at $25{ }^{\circ} \mathrm{C}$ on a rotary evaporator. The liquid residue was distilled $\left(15 \mathrm{mbar}, 31-37^{\circ} \mathrm{C}\right.$, keep the receiver in ice water), affording acetic formic anhydride (7.0 g, 65\%) which was stored in the freezer in a sealed vial under Ar. ${ }^{1} \mathrm{H}$ NMR $\left(400 \mathrm{MHz}, \mathrm{C}_{6} \mathrm{D}_{6}\right) 8.55$ (s, 1H), 1.28 (s 3H). ${ }^{13} \mathrm{C}$ NMR (101 MHz, $\left.\mathrm{C}_{6} \mathrm{D}_{6}\right) \delta 167.19,155.23,19.71$. 


\section{GC-TCD analysis}

\section{$\mathrm{CO}_{2}$ detection}

GC analysis was performed with a Fuli GC 9790 II system with TCD detertor. GC condition: TDX-01 $(2 \mathrm{~m}, 3 \mathrm{~mm}, 60-80 \mathrm{mesh})$, heater temperature $=50{ }^{\circ} \mathrm{C}$, oven temperature $=70{ }^{\circ} \mathrm{C}$, detector temperature $(\mathrm{TCD})=100^{\circ} \mathrm{C}$, splitless, injection volume $=1 \mathrm{~mL}$, carrier gas $=$ argon.

Air sample was taken from standard reaction using 1a under condition A. After the reaction finished, it was cooled to room temperature. A $15 \mathrm{~mL}$ Schlenk tube and the autoclave was linked to the vacuum line with a three-way valve. The air in the tube was evacuated. Then releasing the gas accumulated in the autoclave by carefully open the valve the autoclave. $1 \mathrm{~mL}$ of gas sample was taken from the Schlenk tube via a syringe and subjected to analysis directly. GC graphic was shown in Fig S1.

\section{Figure S1. Detection of $\mathrm{CO}_{2}$ by GC-TCD}

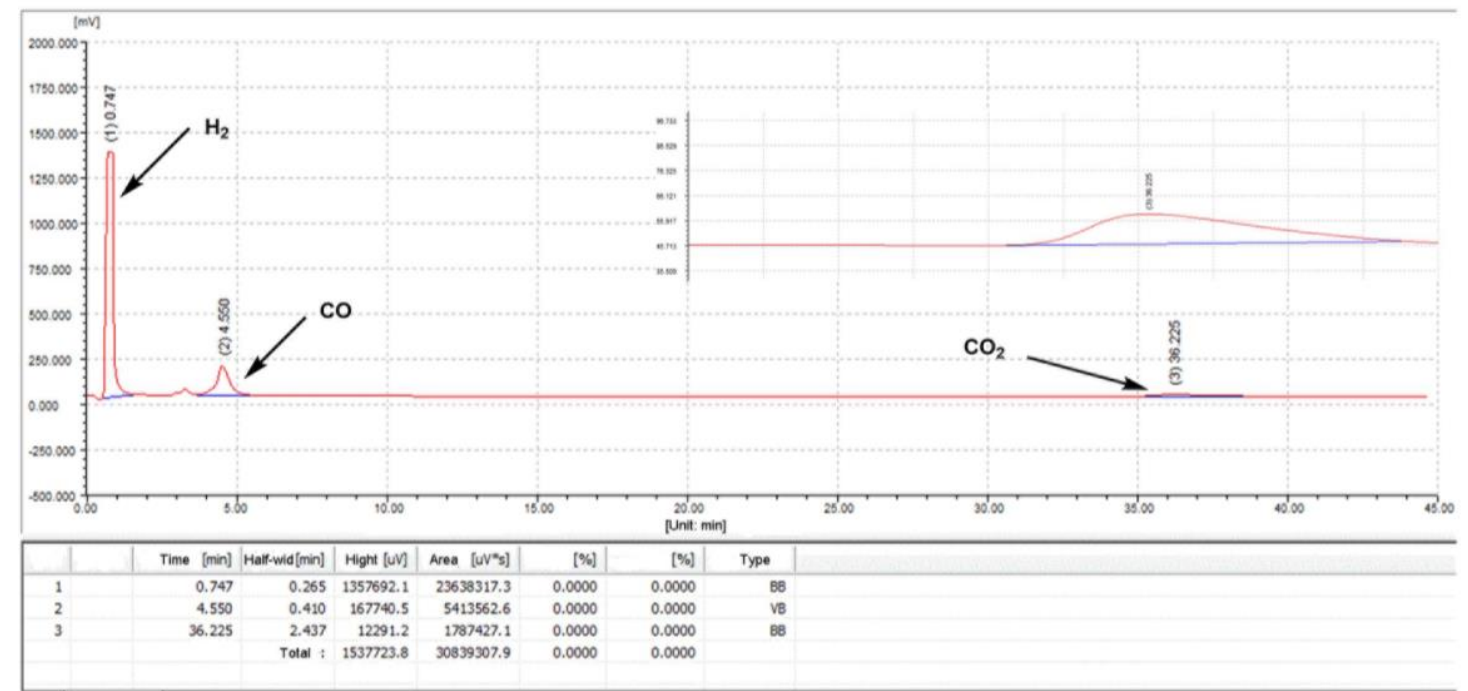




\section{Product characterization}

Unless mentioned seperately, products are purified by chromatogarphy eluting with petroluem and ethyl acetate $(95 / 5, \mathrm{v} / \mathrm{v})$.

(E)-2,3-diphenylacrylaldehyde $(2 a)^{10}$<smiles>O=C/C(=C/c1ccccc1)c1ccccc1</smiles>

$86.3 \mathrm{mg}, 83 \%$ yield (with condition A); $88.4 \mathrm{mg}, 85 \%$ yield (with condition B). White crystal. MP: 89-90 ${ }^{\circ} \mathrm{C} .{ }^{1} \mathrm{H}$ NMR $\left(400 \mathrm{MHz}, \mathrm{CDCl}_{3}\right) \delta 9.77(\mathrm{~s}, 1 \mathrm{H}), 7.47-7.33(\mathrm{~m}, 4 \mathrm{H})$, 7.32 - 7.27 (m, 1H), $7.25-7.17$ (m, 6H). ${ }^{13} \mathrm{C} \mathrm{NMR}\left(101 \mathrm{MHz}, \mathrm{CDCl}_{3}\right) \delta 193.9,150.2$, $141.8,134.0,133.3,130.8,130.2,129.3,128.9,128.5,128.3$.

\section{(E)-2,3-di-p-tolylacrylaldehyde $(2 \mathbf{b})^{10}$}<smiles>Cc1ccc(/C=C(/C=O)c2ccc(C)cc2)cc1</smiles>

$97.9 \mathrm{mg}, 83 \%$ yield (with condition B), white solid. MP: $103-104{ }^{\circ} \mathrm{C} .{ }^{1} \mathrm{H}$ NMR (400 $\left.\mathrm{MHz}, \mathrm{CDCl}_{3}\right) \delta 9.74(\mathrm{~s}, 1 \mathrm{H}), 7.33(\mathrm{~s}, 1 \mathrm{H}), 7.22(\mathrm{~d}, J=7.8 \mathrm{~Hz}, 2 \mathrm{H}), 7.16-7.02(\mathrm{~m}, 6 \mathrm{H})$, 2.39 (s, 3H), 2.31 (s, 3H). ${ }^{13} \mathrm{C}$ NMR (101 MHz, $\left.\mathrm{CDCl}_{3}\right) \delta 194.2,150.1,141.0,140.7$, 138.0, 131.4, 130.8, 130.5, 129.6, 129.3, 129.2, 21.5, 21.4 .

(E)-2,3-di-m-tolylacrylaldehyde $(2 c)^{10}$<smiles>Cc1cccc(/C=C(/C=O)c2cccc(C)c2)c1</smiles>

$87.3 \mathrm{mg}, 74 \%$ yield (with condition B), yellow liquid. ${ }^{1} \mathrm{H} \mathrm{NMR}\left(400 \mathrm{MHz}, \mathrm{CDCl}_{3}\right.$ ): $\delta 9.74(\mathrm{~s}, 1 \mathrm{H}), 7.33(\mathrm{~s}, 1 \mathrm{H}), 7.29-7.27(\mathrm{~m}, 1 \mathrm{H}), 7.20-7.18(\mathrm{~m}, 1 \mathrm{H}), 7.10-6.96(\mathrm{~m}$, $6 \mathrm{H}), 2.35$ (s, 3H), 2.23 (s, 3H). ${ }^{13} \mathrm{C}$ NMR (101 MHz, $\left.\mathrm{CDCl}_{3}\right): \delta$ 194.1, 150.3, 141.9, 
138.5, 138.1, 134.0, 133.4, 131.8, 131.1, 129.8, 129.1, 128.8, 128.4, 127.8, 126.3, 21.5, 21.3 .

(E)-2,3-di-o-tolylacrylaldehyde $(2 d)^{10}$<smiles>Cc1ccccc1/C=C(/C=O)c1ccccc1C</smiles>

$66.0 \mathrm{mg}, 56 \%$ yield (with condition B), colorless liquid. ${ }^{1} \mathrm{H} \mathrm{NMR}\left(400 \mathrm{MHz}, \mathrm{CDCl}_{3}\right)$ : $\delta 9.82(\mathrm{~s}, 1 \mathrm{H}), 7.72(\mathrm{~s}, 1 \mathrm{H}), 7.26-7.15(\mathrm{~m}, 5 \mathrm{H}), 7.00-6.98(\mathrm{~m}, 1 \mathrm{H}), 6.89-6.85(\mathrm{~m}$, $1 \mathrm{H}), 6.79-6.75(\mathrm{~m}, 1 \mathrm{H}), 2.50(\mathrm{~s}, 3 \mathrm{H}), 2.03(\mathrm{~s}, 3 \mathrm{H}) .{ }^{13} \mathrm{C} \mathrm{NMR}\left(101 \mathrm{MHz}, \mathrm{CDCl}_{3}\right): \delta$ $193.9,148.0,142.3,138.0,136.3,133.1,130.6,130.4,129.9,129.6,129.0,128.4$, 126.2, 125.9, 20.1, 19.6.

(E)-2,3-bis(4-methoxyphenyl)acrylaldehyde $(2 \mathrm{e})^{10}$<smiles>COc1ccc(/C=C(/C=O)c2ccc(OC)cc2)cc1</smiles>

$107.2 \mathrm{mg}, 80 \%$ yield (with condition B), white solid. MP: $98-99{ }^{\circ} \mathrm{C} .{ }^{1} \mathrm{H}$ NMR (400 $\left.\mathrm{MHz}, \mathrm{CDCl}_{3}\right): \delta 9.69(\mathrm{~s}, 1 \mathrm{H}), 7.28(\mathrm{~s}, 1 \mathrm{H}), 7.21-7.19(\mathrm{~m}, 2 \mathrm{H}), 7.14-7.12(\mathrm{~m}, 2 \mathrm{H})$, $6.96-6.94(\mathrm{~m}, 2 \mathrm{H}), 6.77-6.75(\mathrm{~m}, 2 \mathrm{H}), 3.84(\mathrm{~s}, 3 \mathrm{H}), 3.78(\mathrm{~s}, 3 \mathrm{H}) .{ }^{13} \mathrm{C}$ NMR $(101$ $\left.\mathrm{MHz}, \mathrm{CDCl}_{3}\right): \delta 194.3,161.2,159.5,150.1,139.5,132.6,130.6,126.9,125.7,114.5$, 114.0, 55.3, 55.3.

(E)-2,3-bis(3-methoxyphenyl)acrylaldehyde $(2 \mathrm{f})^{10}$<smiles>COc1cccc(C=C(C)c2cccc(OC)c2)c1</smiles> 
$103.2 \mathrm{mg}, 77 \%$ yield (with condition B), white solid. MP: $48-50{ }^{\circ} \mathrm{C} .{ }^{1} \mathrm{H}$ NMR (400 $\left.\mathrm{MHz}, \mathrm{CDCl}_{3}\right): \delta 9.72(\mathrm{~s}, 1 \mathrm{H}), 7.36-7.28(\mathrm{~m}, 2 \mathrm{H}), 7.16(\mathrm{t}, J=8.0 \mathrm{~Hz}, 1 \mathrm{H}), 6.94-6.89$ $(\mathrm{m}, 1 \mathrm{H}), 6.88-6.81(\mathrm{~m}, 2 \mathrm{H}), 6.80-6.76(\mathrm{~m}, 1 \mathrm{H}), 6.75-6.69(\mathrm{~m}, 2 \mathrm{H}), 3.74(\mathrm{~s}, 3 \mathrm{H})$, $3.51(\mathrm{~s}, 3 \mathrm{H}) .{ }^{13} \mathrm{C} \mathrm{NMR}\left(101 \mathrm{MHz}, \mathrm{CDCl}_{3}\right) \delta 193.8,160.0,159.3,150.1,141.8,135.2$, $134.9,130.1,129.5,123.9,121.6,117.2,114.7,114.6,114.1,55.3,54.9$.

(E)-2,3-bis(2-methoxyphenyl)acrylaldehyde $(2 \mathrm{~g})^{10}$<smiles>COc1ccccc1/C=C(/C=O)c1ccccc1OC</smiles>

$105.8 \mathrm{mg}, 79 \%$ yield (with condition B), colorless liquid. ${ }^{1} \mathrm{H}$ NMR (400 MHz, $\mathrm{CDCl}_{3}$ ): $\delta 9.76(\mathrm{~s}, 1 \mathrm{H}), 7.93(\mathrm{~s}, 1 \mathrm{H}), 7.33(\mathrm{ddd}, J=8.2,7.3,1.9 \mathrm{~Hz}, 1 \mathrm{H}), 7.24(\mathrm{ddd}, J=8.3,7.5$, $1.7 \mathrm{~Hz}, 1 \mathrm{H}), 7.04-6.84(\mathrm{~m}, 5 \mathrm{H}), 6.63$ (t, $J=7.6 \mathrm{~Hz}, 1 \mathrm{H}), 3.87$ (s, 3H), 3.67 (s, 3H). ${ }^{13} \mathrm{C}$ NMR (101 MHz, $\left.\mathrm{CDCl}_{3}\right) \delta 193.9,158.0,157.1,144.5,138.7,131.5,130.7,129.8$, $129.8,123.5,123.2,121.1,120.2,111.4,110.7,55.7,55.6$.

(E)-2,3-bis(4-fluorophenyl)acrylaldehyde $(2 \mathrm{~h})^{10}$<smiles>O=C/C(=C/c1ccc(F)cc1)c1ccc(F)cc1</smiles>

$65.8 \mathrm{mg}, 54 \%$ yield (with condition B), yellow solid. MP: $109-110{ }^{\circ} \mathrm{C} .{ }^{1} \mathrm{H}$ NMR (400 $\left.\mathrm{MHz} \mathrm{CDCl}_{3}\right): \delta 9.74(\mathrm{~s}, 1 \mathrm{H}), 7.36(\mathrm{~s}, 1 \mathrm{H}), 7.23-7.12(\mathrm{~m}, 6 \mathrm{H}), 6.95(\mathrm{t}, J=8.5 \mathrm{~Hz}$, 2H). ${ }^{13} \mathrm{C}$ NMR (101 MHz, $\left.\mathrm{CDCl}_{3}\right): \delta$ 193.6, 164.9, 164.0, 162.4, 161.5, 149.2, 140.5, $132.8,132.7,131.3,131.2,130.1,130.1,128.8,116.3,116.0,116.0,115.8$.

(E)-2,3-bis(4-chlorophenyl)acrylaldehyde (2i) $)^{10}$ 
<smiles>O=C/C(=C/c1ccc(Cl)cc1)c1ccc(Cl)cc1</smiles>

$56.6 \mathrm{mg}, 41 \%$ yield (with condition B), yellow solid. MP: $106-107{ }^{\circ} \mathrm{C} .{ }^{1} \mathrm{H}$ NMR (400 $\left.\mathrm{MHz}, \mathrm{CDCl}_{3}\right): \delta 9.73(\mathrm{~s}, 1 \mathrm{H}), 7.40-7.38(\mathrm{~m}, 2 \mathrm{H}), 7.35(\mathrm{~s}, 1 \mathrm{H}), 7.24-7.22(\mathrm{~m}, 2 \mathrm{H})$, $7.15-7.11(\mathrm{~m}, 4 \mathrm{H}) .{ }^{13} \mathrm{C} \mathrm{NMR}\left(101 \mathrm{MHz}, \mathrm{CDCl}_{3}\right): \delta 193.1,148.9,140.9,136.5,134.6$, $132.2,131.8,131.3,130.8,129.3,129.0$.

(E)-2,3-bis(4-bromophenyl)acrylaldehyde $(2 \mathbf{j})^{10}$<smiles>O=C/C(=C/c1ccc(Br)cc1)c1ccc(Br)cc1</smiles>

$80.0 \mathrm{mg}, 44 \%$ yield (with condition B), white solid. MP: $109-110{ }^{\circ} \mathrm{C} .{ }^{1} \mathrm{H}$ NMR (400 $\left.\mathrm{MHz}, \mathrm{CDCl}_{3}\right): \delta 9.73(\mathrm{~s}, 1 \mathrm{H}), 7.54(\mathrm{~d}, J=8.4 \mathrm{~Hz}, 2 \mathrm{H}), 7.39(\mathrm{~d}, J=8.5 \mathrm{~Hz}, 2 \mathrm{H}), 7.33$ (s, 1H), 7.06 (dd, $J=8.4,3.3 \mathrm{~Hz}, 4 \mathrm{H}) .{ }^{13} \mathrm{C} \mathrm{NMR}\left(101 \mathrm{MHz}, \mathrm{CDCl}_{3}\right): \delta$ 193.1, 148.9, 141.0, 132.6, 132.2, 132.0, 131.9, 131.7, 131.1, 124.9, 122.9.

(E)-2,3-di(naphthalen-1-yl)acrylaldehyde (2l $)^{11}$<smiles>O=C/C(=C/c1cccc2ccccc12)c1cccc2ccccc12</smiles>

112.4 mg, 73\% yield (with condition B), white solid. Products was eluted with PE and $\operatorname{DCM}(8 / 1, \mathrm{v} / \mathrm{v}) .{ }^{1} \mathrm{H}$ NMR $\left(400 \mathrm{MHz}, \mathrm{CDCl}_{3}\right) \delta 10.10(\mathrm{~s}, 1 \mathrm{H}), 8.52(\mathrm{~s}, 1 \mathrm{H}), 8.29(\mathrm{~d}, J=$ $8.4 \mathrm{~Hz}, 1 \mathrm{H}), 7.88-7.81$ (m, 3H), $7.71-7.62$ (m, 3H), 7.55 (ddd, $J=8.0,6.8,1.2 \mathrm{~Hz}$, $1 \mathrm{H}), 7.47-7.33(\mathrm{~m}, 3 \mathrm{H}), 7.21(\mathrm{dd}, J=7.0,1.2 \mathrm{~Hz}, 1 \mathrm{H}), 6.99-6.93(\mathrm{~m}, 2 \mathrm{H}) .{ }^{13} \mathrm{C} \mathrm{NMR}$ $\left(101 \mathrm{MHz}, \mathrm{CDCl}_{3}\right) \delta 193.7,148.1,142.5,133.7,133.5,131.7,131.6,131.5,130.5$, 
$130.4,129.0,128.8,128.6,127.9,127.6,127.1,126.5,126.3,126.0,125.6,125.1,125.0$, 123.2.

(E)-2,3-di(naphthalen-2-yl)acrylaldehyde (2m) $)^{11}$<smiles>O=C/C(=C/c1ccc2ccccc2c1)c1ccc2ccccc2c1</smiles>

$101.6 \mathrm{mg}$, 66\% yield (80\% NMR yield, with condition B), white solid. Products was eluted with PE and DCM (8/1, v/v). ${ }^{1} \mathrm{H}$ NMR $\left(400 \mathrm{MHz}, \mathrm{CDCl}_{3}\right) \delta 9.87(\mathrm{~s}, 1 \mathrm{H}), 7.88-$ $7.77(\mathrm{~m}, 5 \mathrm{H}), 7.68(\mathrm{td}, J=7.0,3.6 \mathrm{~Hz}, 2 \mathrm{H}), 7.60(\mathrm{~s}, 1 \mathrm{H}), 7.53-7.39(\mathrm{~m}, 5 \mathrm{H}), 7.28(\mathrm{dd}$, $J=8.4,1.7 \mathrm{~Hz}, 1 \mathrm{H}), 7.13(\mathrm{dd}, J=8.7,1.8 \mathrm{~Hz}, 1 \mathrm{H}) .{ }^{13} \mathrm{C} \mathrm{NMR}\left(101 \mathrm{MHz}, \mathrm{CDCl}_{3}\right) \delta$ 193.9, 150.4, 141.5, 133.9, 133.5, 133.2, 133.0, 132.3, 131.8, 130.8, 129.0, 128.7, 128.5, $128.4,128.0,127.8,127.7,127.3,126.7,126.5,126.5,126.2$.

(E)-3-(benzo $[d][1,3]$ dioxol-4-yl)-2-(benzo $[d][1,3]$ dioxol-5-yl)acrylaldehyde(2n)<smiles>C/C(=C/c1cccc2c1OCO2)c1ccc2c(c1)OCO2</smiles>

$113.9 \mathrm{mg}, 77 \%$ yield (with condition B), yellow solid. Products was eluted with PE and EA (10/1, v/v). MP: $141-142{ }^{\circ} \mathrm{C} .{ }^{1} \mathrm{H}$ NMR $\left(400 \mathrm{MHz}, \mathrm{CDCl}_{3}\right) \delta 9.66(\mathrm{~s}, 1 \mathrm{H}), 7.24(\mathrm{~s}$, $1 \mathrm{H}), 6.94-6.89(\mathrm{~m}, 1 \mathrm{H}), 6.81(\mathrm{dd}, J=45.6,8.2 \mathrm{~Hz}, 2 \mathrm{H}), 6.67-6.62(\mathrm{~m}, 3 \mathrm{H}), 6.00(\mathrm{~s}$, $3 \mathrm{H}), 5.95(\mathrm{~s}, 3 \mathrm{H}) .{ }^{13} \mathrm{C} \mathrm{NMR}\left(101 \mathrm{MHz}, \mathrm{CDCl}_{3}\right) \delta 193.9,150.1,149.6,148.2,147.8$, 147.7, 139.7, 128.3, 127.4, 126.8, 122.9, 109.7, 109.6, 109.1, 108.5, 101.6, 101.2. HRMS (ESI) m/z: [M+Na] ${ }^{+}$Calcd for $\mathrm{C}_{17} \mathrm{H}_{12} \mathrm{NaO}_{5} 319.0582$; Found 319.0581.

(E)-2,3-di(furan-3-yl)acrylaldehyde(2o)<smiles>O=C/C(=C/c1ccoc1)c1ccoc1</smiles> 
$70.5 \mathrm{mg}, 75 \%$ yield (with condition B), yellow liquid. ${ }^{1} \mathrm{H} \mathrm{NMR}\left(400 \mathrm{MHz}, \mathrm{CDCl}_{3}\right) \delta$ $9.60(\mathrm{~s}, 1 \mathrm{H}), 7.73-7.68(\mathrm{~m}, 1 \mathrm{H}), 7.58(\mathrm{dd}, J=1.5,0.8 \mathrm{~Hz}, 1 \mathrm{H}), 7.50$ (t, $J=1.7 \mathrm{~Hz}$, $1 \mathrm{H}), 7.37-7.35(\mathrm{~m}, 1 \mathrm{H}), 7.24(\mathrm{~s}, 1 \mathrm{H}), 6.42-6.36(\mathrm{~m}, 2 \mathrm{H}) .{ }^{13} \mathrm{C} \mathrm{NMR}\left(101 \mathrm{MHz}, \mathrm{CDCl}_{3}\right)$ $\delta 192.7,146.2,143.9,142.8,142.2,140.5,132.2,121.7,116.2,111.1,109.9$. HRMS (ESI) $\mathrm{m} / \mathrm{z}:[\mathrm{M}+\mathrm{Na}]^{+}$Calcd for $\mathrm{C}_{11} \mathrm{H}_{8} \mathrm{NaO}_{3}$ 211.0371; Found 211.0371.

\section{Cinnamaldehyde $(2 p)^{12}$}

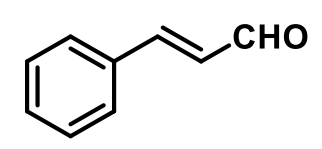

$5.9 \mathrm{mg}, 9 \%$ yield (with condition B), pale-yellow liquid. $36 \mathrm{mg}(70 \%)$ styrene was obtained as by-product. ${ }^{1} \mathrm{H}$ NMR $\left(400 \mathrm{MHz}, \mathrm{CDCl}_{3}\right) \delta 9.71(\mathrm{~d}, \mathrm{~J}=7.8 \mathrm{~Hz}, 1 \mathrm{H}), 7.60-$ 7.55 (m, 2H), 7.49 (d, J = 15.9 Hz, 1H), $7.46-7.43$ (m, 3H), 6.73 (dd, J = 16.0, 7.7 Hz, 1H) ${ }^{13} \mathrm{C}$ NMR $\left(101 \mathrm{MHz}, \mathrm{CDCl}_{3}\right) \delta 193.8,152.8,133.9,131.3,129.1,128.6,128.5$.

(E)-2-methylbut-2-enal (2q) $)^{12}$

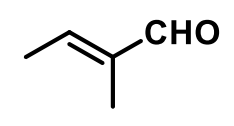

$61 \%$ GC yield (with condition B), pale-yellow liquid. ${ }^{1} \mathrm{H}$ NMR (400 $\left.\mathrm{MHz}, \mathrm{CDCl}_{3}\right) \delta$ $9.41(\mathrm{~s}, 1 \mathrm{H}), 6.61(\mathrm{q}, J=7.0 \mathrm{~Hz}, 1 \mathrm{H}), 1.99(\mathrm{dd}, J=7.0,1.1 \mathrm{~Hz}, 3 \mathrm{H}), 1.76(\mathrm{~s}, 1 \mathrm{H}) .{ }^{13} \mathrm{C}$ NMR (101 MHz, $\left.\mathrm{CDCl}_{3}\right) \delta 195.2,149.6,140.4,14.9,8.9$.

(E)-2-butylhept-2-enal(2r) ${ }^{11}$

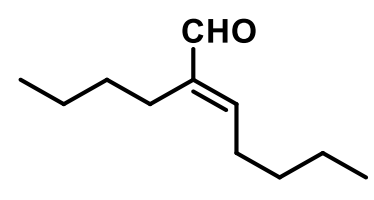

$57.1 \mathrm{mg}, 68 \%$ yield (with condition B), colorless liquid. ${ }^{1} \mathrm{H} \mathrm{NMR}\left(400 \mathrm{MHz}, \mathrm{CDCl}_{3}\right) \delta$ $9.36(\mathrm{~s}, 1 \mathrm{H}), 6.45(\mathrm{t}, J=7.4 \mathrm{~Hz}, 1 \mathrm{H}), 2.40-2.32$ (q, $J=7.4 \mathrm{~Hz} 2 \mathrm{H}), 2.26-2.20(\mathrm{~m}$, $2 \mathrm{H}), 1.59-1.20(\mathrm{~m}, 8 \mathrm{H}), 1.07-0.85(\mathrm{~m}, 6 \mathrm{H}) .{ }^{13} \mathrm{C} \mathrm{NMR}\left(101 \mathrm{MHz}, \mathrm{CDCl}_{3}\right) \delta 195.3$, 155.3, 143.8, 30.9, 30.8, 28.6, 23.7, 22.7, 22.4, 13.8, 13.8 .

(E)-2-hexylnon-2-enal $(2 s)^{13}$ 


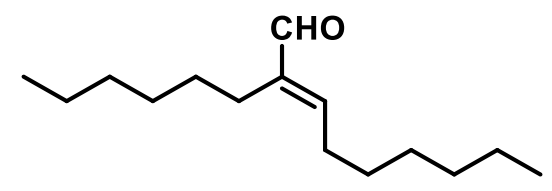

$96.3 \mathrm{mg}, 86 \%$ yield (with condition B), colorless liquid. ${ }^{1} \mathrm{H}$ NMR (400 $\left.\mathrm{MHz}, \mathrm{CDCl}_{3}\right) \delta$ 9.29 (s, 1H), 6.37 (t, $J=7.4 \mathrm{~Hz}, 1 \mathrm{H}), 2.28(\mathrm{q}, J=7.4 \mathrm{~Hz}, 2 \mathrm{H}), 2.15(\mathrm{t}, J=7.1 \mathrm{~Hz}, 2 \mathrm{H})$, $1.42(\mathrm{q}, J=7.3 \mathrm{~Hz}, 2 \mathrm{H}), 1.30-1.16(\mathrm{~m}, 14 \mathrm{H}), 0.85-0.75(\mathrm{~m}, 6 \mathrm{H}) .{ }^{13} \mathrm{C}$ NMR $(101$ $\left.\mathrm{MHz}, \mathrm{CDCl}_{3}\right) \delta 195.2,155.3,143.8,31.6,31.6,29.3,29.1,28.9,28.7,28.6,24.0,22.58$, $22.5,14.0,14.0$.

(E)-cyclododec-1-ene-1-carbaldehyde $(2 \mathrm{t})^{13}$<smiles>O=CC1=CCCCCCCCCCC1</smiles>

$75.6 \mathrm{mg}, 78 \%$ yield (with condition B), pale yellow liquid. ${ }^{1} \mathrm{H} \mathrm{NMR}\left(400 \mathrm{MHz}, \mathrm{CDCl}_{3}\right)$ $\delta 9.35(\mathrm{~s}, 1 \mathrm{H}), 6.45$ (t, $J=8.1 \mathrm{~Hz}, 1 \mathrm{H}), 2.35$ (q, $J=7.5 \mathrm{~Hz}, 2 \mathrm{H}), 2.28$ (t, $J=6.7 \mathrm{~Hz}$, $2 \mathrm{H}), 1.62-1.50(\mathrm{~m}, 4 \mathrm{H}), 1.42-1.29(\mathrm{~m}, 10 \mathrm{H}), 1.18-1.12(\mathrm{~m}, 2 \mathrm{H}) .{ }^{13} \mathrm{C}$ NMR (101 $\left.\mathrm{MHz}, \mathrm{CDCl}_{3}\right) \delta 195.9,155.7,143.4,26.2,25.8,25.7,25.2,25.1,24.8,23.4,22.8,22.02$, 21.4 .

(E)-2-ethylideneheptanal (2u) and (E)-2-methyloct-2-enal (minor) ${ }^{14}$<smiles>C/C=C(/C=O)CCCCC</smiles>

$52.5 \mathrm{mg}, 75 \%$ yield, $r r=50: 25$ (with condition B), pale yellow liquid. ${ }^{1} \mathrm{H}$ NMR (400 $\left.\mathrm{MHz}, \mathrm{CDCl}_{3}\right) \delta 9.40(\mathrm{~s}, 1 \mathrm{H}), 9.36(\mathrm{~s}, 0.53 \mathrm{H}), 6.56(\mathrm{q}, J=7.0 \mathrm{~Hz}, 0.63 \mathrm{H}$, minor), 6.50 $(\mathrm{td}, J=7.4,1.4 \mathrm{~Hz}, 1 \mathrm{H}), 2.35(\mathrm{q}, J=7.4 \mathrm{~Hz}, 2.2 \mathrm{H}), 2.25$ (t, $J=7.5 \mathrm{~Hz}, 1.2 \mathrm{H}), 1.99$ (d, $J=7.0 \mathrm{~Hz}, 1.7 \mathrm{H}$, minor), $1.74(\mathrm{~s}, 3 \mathrm{H}), 1.56-1.46(\mathrm{~m}, 2.3 \mathrm{H}), 1.39-1.22(\mathrm{~m}, 9 \mathrm{H}), 0.93$ - 0.85 (m, 5H). ${ }^{13} \mathrm{C}$ NMR (101 MHz, $\left.\mathrm{CDCl}_{3}\right) \delta 195.4,195.1$ (minor), 155.0, 149.7 (minor), 145.0 (minor), 139.3, 31.8, 31.5, 28.9, 28.1, 28.1, 23.6, 22.5, 22.5, 14.8, 14.0, $13.9,9.2$. 
(E)-3-cyclohexyl-2-methylacrylaldehyde (2v) and (E)-2-cyclohexylbut-2-enal (minor) ${ }^{15,16}$

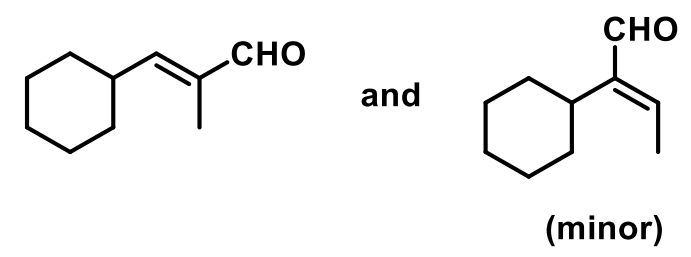

$53.9 \mathrm{mg}, 71 \%$ yield, $r r=58: 13$ (with condition B), pale yellow liquid. ${ }^{1} \mathrm{H}$ NMR $(400$ $\left.\mathrm{MHz}, \mathrm{CDCl}_{3}\right) \delta 9.37(\mathrm{~s}, 1 \mathrm{H}), 9.31(\mathrm{~s}, 0.22 \mathrm{H}$, minor), $6.46(\mathrm{q}, J=7.1 \mathrm{~Hz}, 0.23 \mathrm{H}$, minor), $6.30(\mathrm{dd}, J=9.5,1.4 \mathrm{~Hz}, 1 \mathrm{H}), 2.58-2.47(\mathrm{~m}, 1.25 \mathrm{H}), 2.01(\mathrm{~d}, J=7.2 \mathrm{~Hz}, 0.68 \mathrm{H}$, minor), $1.85-1.63(\mathrm{~m}, 9 \mathrm{H}), 1.48-1.13(\mathrm{~m}, 7 \mathrm{H}) .{ }^{13} \mathrm{C} \mathrm{NMR}\left(101 \mathrm{MHz}, \mathrm{CDCl}_{3}\right) \delta 195.7$, 195.5 (minor), 159.7, 150.2 (minor), 148.2 (minor), 137.4, 37.9, 36.4, 32.8, 32.6, 31.7, 29.7, 26.9, 26.7, 25.9, 25.8, 25.4, 15.0, 9.2.

(E)-2-methyl-3-phenylacrylaldehyde (2w) and $(E)$-2-phenylbut-2-enal (minor) $)^{12}$<smiles>CC(C=O)=Cc1ccccc1</smiles>
and<smiles>C/C=C(/C=O)c1ccccc1</smiles>

$62.4 \mathrm{mg}, 80 \%$ yield, $r r=65: 15$ (with condition B), pale yellow liquid. ${ }^{1} \mathrm{H}$ NMR (400 $\left.\mathrm{MHz}, \mathrm{CDCl}_{3}\right) \delta 9.58(\mathrm{~s}, 1 \mathrm{H}), 7.57-7.36(\mathrm{~m}, 5 \mathrm{H}), 7.26(\mathrm{~s}, 1 \mathrm{H}), 2.07(\mathrm{~s}, 3 \mathrm{H}) .{ }^{13} \mathrm{C} \mathrm{NMR}$ $\left(101 \mathrm{MHz}, \mathrm{CDCl}_{3}\right) \delta 195.7,149.9,138.3,135.1,130.0,129.6,128.7,10.9$. Minor isomer is unable to be separated with other by-products.

(E)-2-butylhept-2-enal $(2 x)^{13}$<smiles>CCCC/C(C=O)=C\c1ccccc1</smiles>

and<smiles>CCCC/C=C(/C=O)c1ccccc1</smiles>

$69.6 \mathrm{mg}, 74 \%$ yield (with condition B), $r r=64: 10$, colorless liquid.

Major. ${ }^{1} \mathrm{H}$ NMR $\left(400 \mathrm{MHz}, \mathrm{CDCl}_{3}\right) \delta 9.54(\mathrm{~s}, 1 \mathrm{H}), 7.56-7.32(\mathrm{~m}, 5 \mathrm{H}), 7.21(\mathrm{~s}, 1 \mathrm{H})$, $2.62-2.41(\mathrm{~m}, 2 \mathrm{H}), 1.58-1.30(\mathrm{~m}, 4 \mathrm{H}), 0.93(\mathrm{t}, J=7.1 \mathrm{~Hz}, 3 \mathrm{H}) .{ }^{13} \mathrm{C}$ NMR $(101 \mathrm{MHz}$, $\left.\mathrm{CDCl}_{3}\right) \delta 195.7,149.8,143.4,134.9,129.66,129.5,128.8,30.4,24.6,23.0,13.8$.

Minor. ${ }^{1} \mathrm{H}$ NMR (400 MHz, $\left.\mathrm{CDCl}_{3}\right) \delta 9.62(\mathrm{~s}, 1 \mathrm{H}), 7.45-7.29(\mathrm{~m}, 3 \mathrm{H}), 7.21-7.07$ $(\mathrm{m}, 2 \mathrm{H}), 6.73$ (t, $J=7.6 \mathrm{~Hz}, 1 \mathrm{H}), 2.37$ (q, $J=7.5 \mathrm{~Hz}, 2 \mathrm{H}), 1.61-1.42(\mathrm{~m}, 2 \mathrm{H}), 1.33$ 
$(\mathrm{dq}, J=14.4,7.2 \mathrm{~Hz}, 2 \mathrm{H}), 0.88(\mathrm{t}, J=7.3 \mathrm{~Hz}, 3 \mathrm{H}) .{ }^{13} \mathrm{C} \mathrm{NMR}\left(101 \mathrm{MHz}, \mathrm{CDCl}_{3}\right) \delta 193.7$, 156.6, 143.9, 132.6, 129.4, 128.2, 127.9, 30.9, 29.5, 22.4, 13.8 .

(Z)-2-(methoxymethyl)-3-phenylprop-2-en-1-ol (2y' $)^{17}$

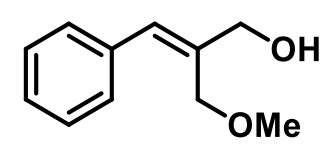

Original aldehyde was in an equilibrium with its acetal form. After removing solvent, the reaction mixture was treated with $\mathrm{NaBH}_{4}$. The aldehyde and acetal were converted to alcohol. Colorless liquid, $72.9 \mathrm{mg}, 82 \%$ yield (with condition B). ${ }^{1} \mathrm{H}$ NMR (400 MHz, $\left.\mathrm{CDCl}_{3}\right) \delta 7.38-7.32(\mathrm{~m}, 2 \mathrm{H}), 7.30-7.20(\mathrm{~m}, 3 \mathrm{H}), 6.72(\mathrm{~s}, 1 \mathrm{H}), 4.33(\mathrm{~s}, 2 \mathrm{H}), 4.20(\mathrm{~s}$, 2H), 3.36 (s, 3H), 2.43 (brs, $1 \mathrm{H}) .{ }^{13} \mathrm{C} \mathrm{NMR}\left(101 \mathrm{MHz}, \mathrm{CDCl}_{3}\right) \delta$ 137.6, 136.3, 129.9, $128.8,128.2,127.2,70.2,66.9,58.6$.

(E)-hept-2-enal $(2 z)^{18}$

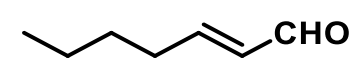

$6.2 \mathrm{mg}, 11 \%$ (NMR yield, with condition B), pale-yellow liquid. 17\% 1-hexene was identified on GC as by-product. ${ }^{1} \mathrm{H}$ NMR $\left(400 \mathrm{MHz}, \mathrm{CDCl}_{3}\right) \delta 9.52(\mathrm{~d}, J=7.9 \mathrm{~Hz}, 1 \mathrm{H})$, $6.87(\mathrm{dt}, J=15.6,6.8 \mathrm{~Hz}, 1 \mathrm{H}), 6.13(\mathrm{dd}, J=15.6,7.9 \mathrm{~Hz}, 1 \mathrm{H}), 2.47-2.18(\mathrm{~m}, 2 \mathrm{H})$, $1.53(\mathrm{p}, J=7.2 \mathrm{~Hz}, 2 \mathrm{H}), 1.40-1.26(\mathrm{~m}, 4 \mathrm{H}), 0.91(\mathrm{t}, J=6.9 \mathrm{~Hz}, 3 \mathrm{H}) .{ }^{13} \mathrm{C} \mathrm{NMR}(101$ $\left.\mathrm{MHz}, \mathrm{CDCl}_{3}\right) \delta 194.2,159.1,132.9,32.7,31.3,27.5,22.4,13.9$. 

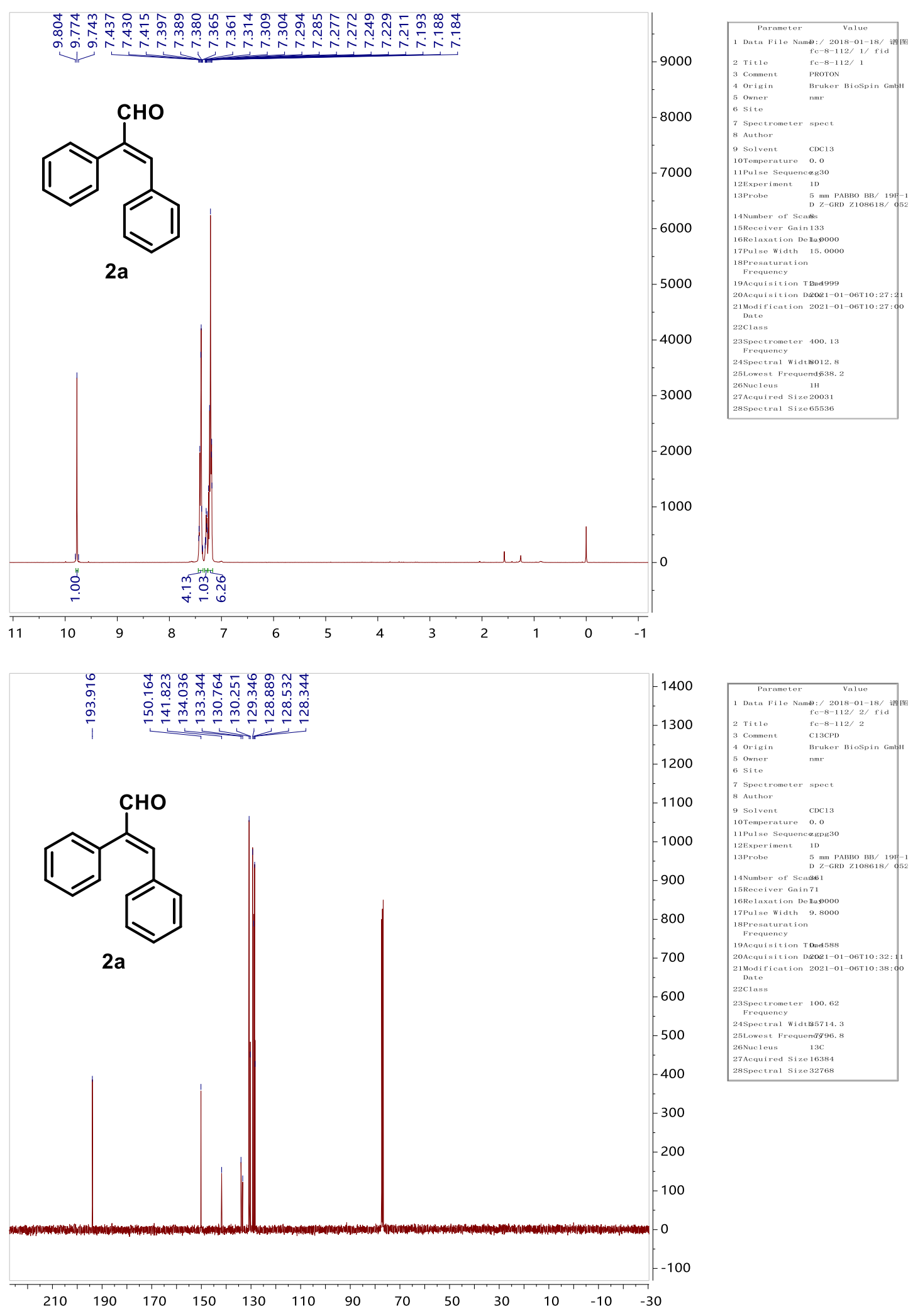


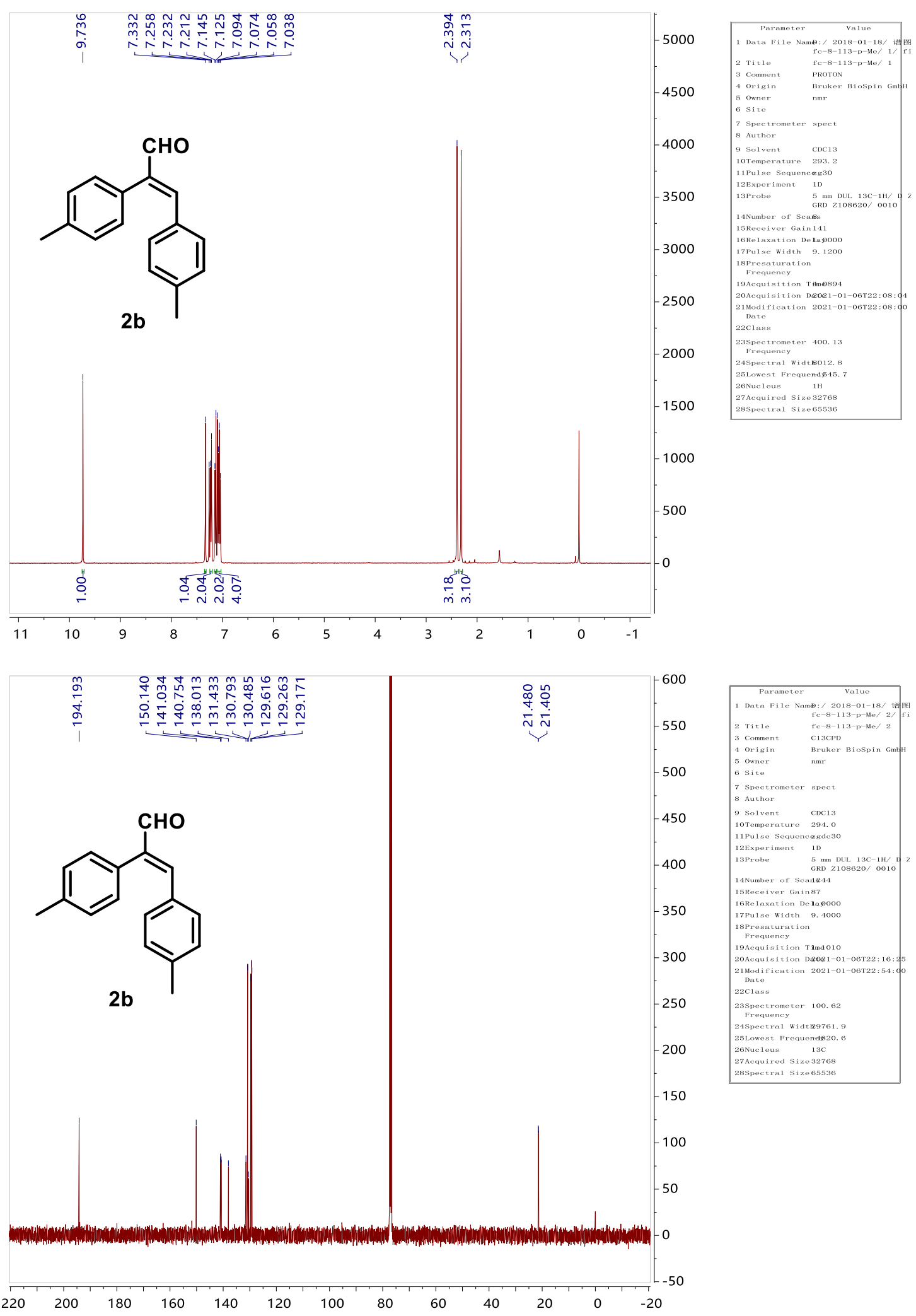




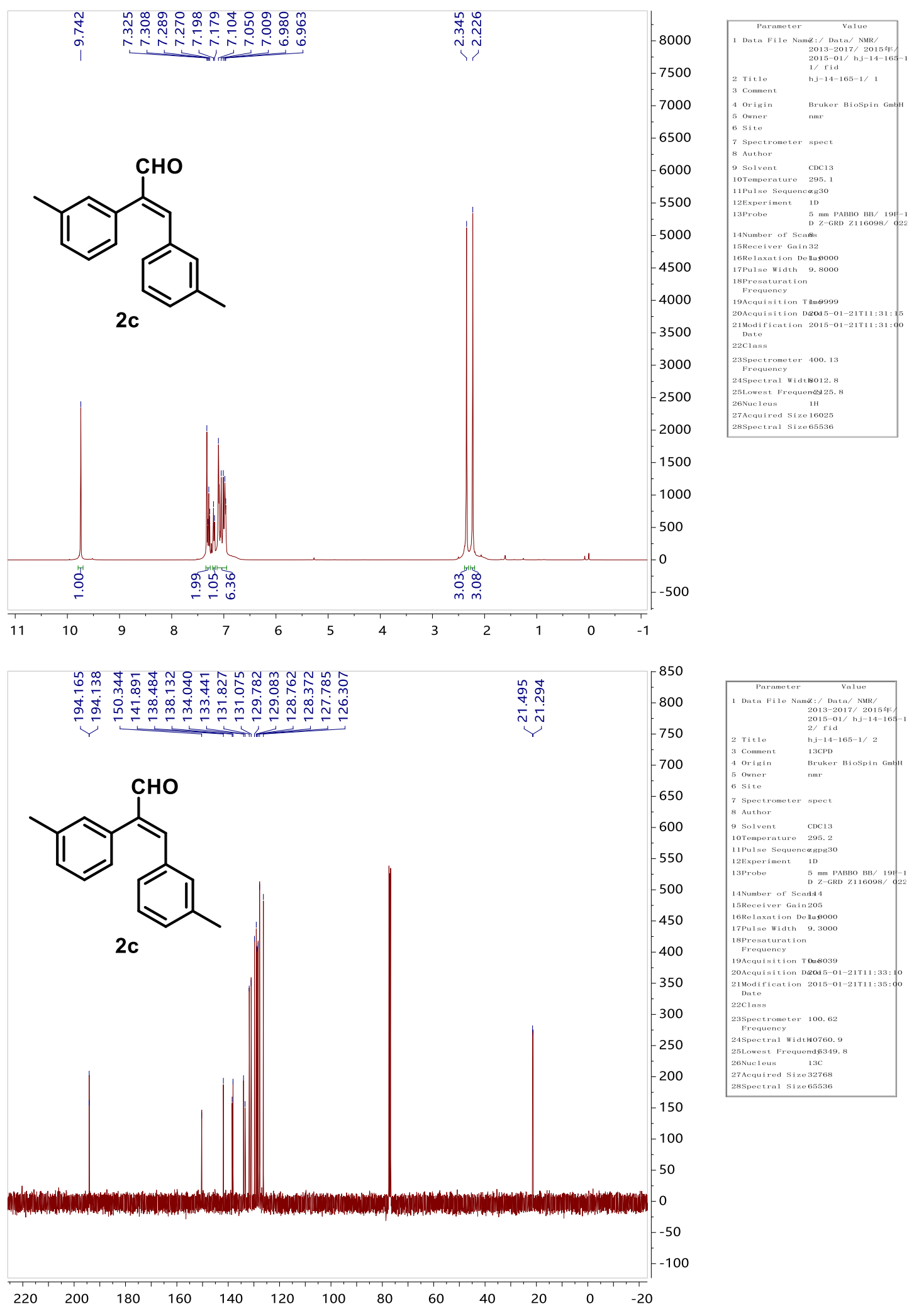




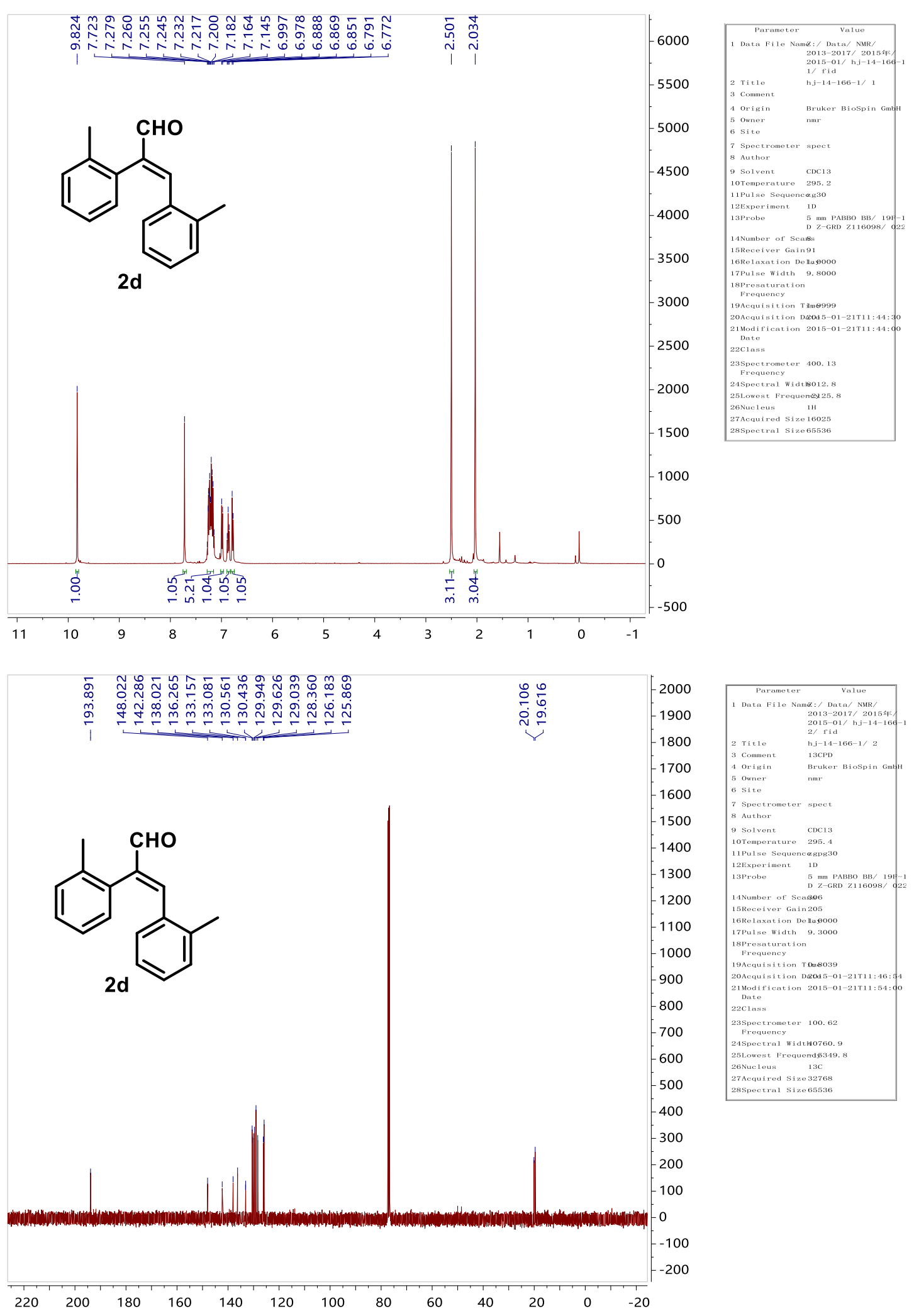




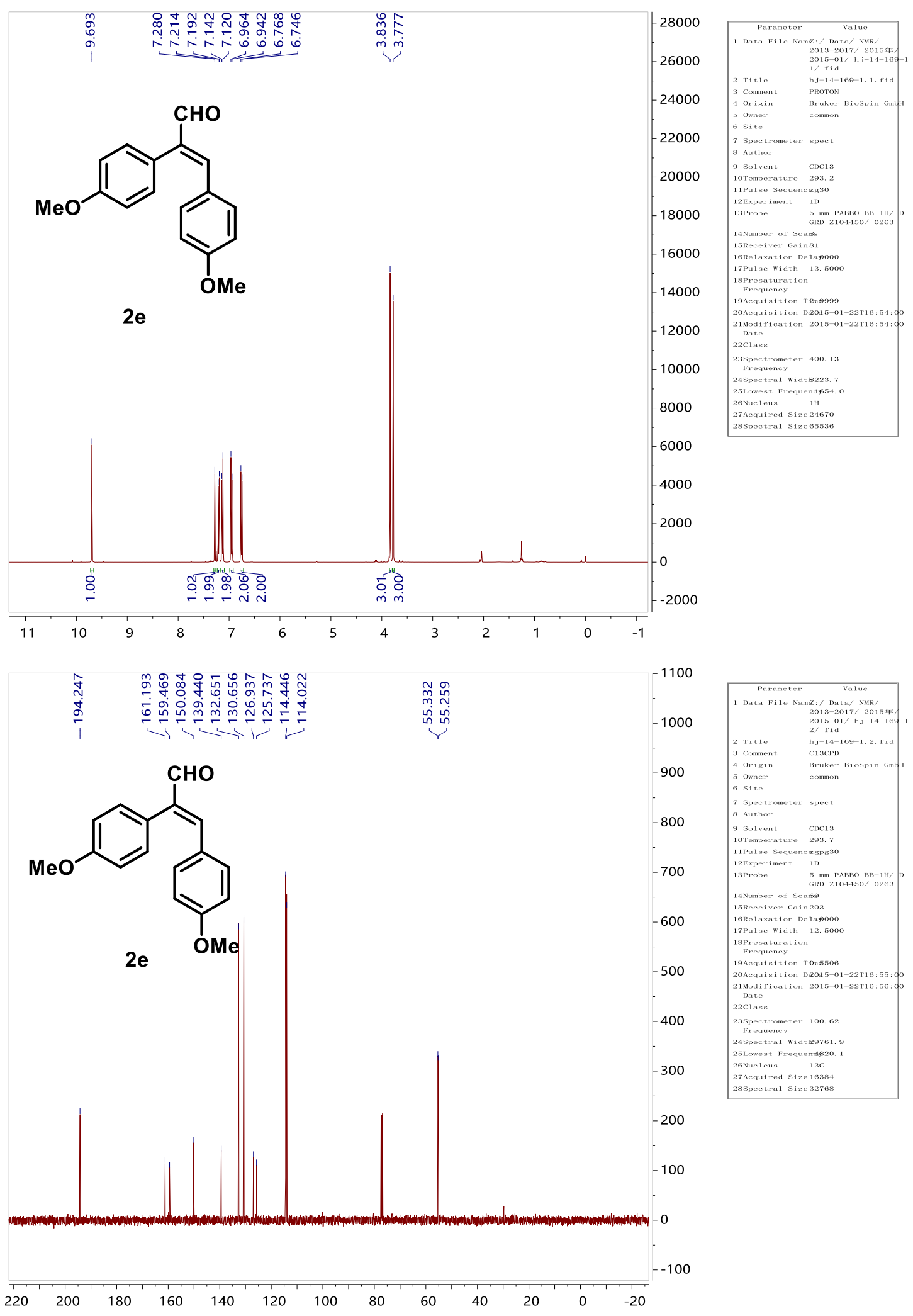



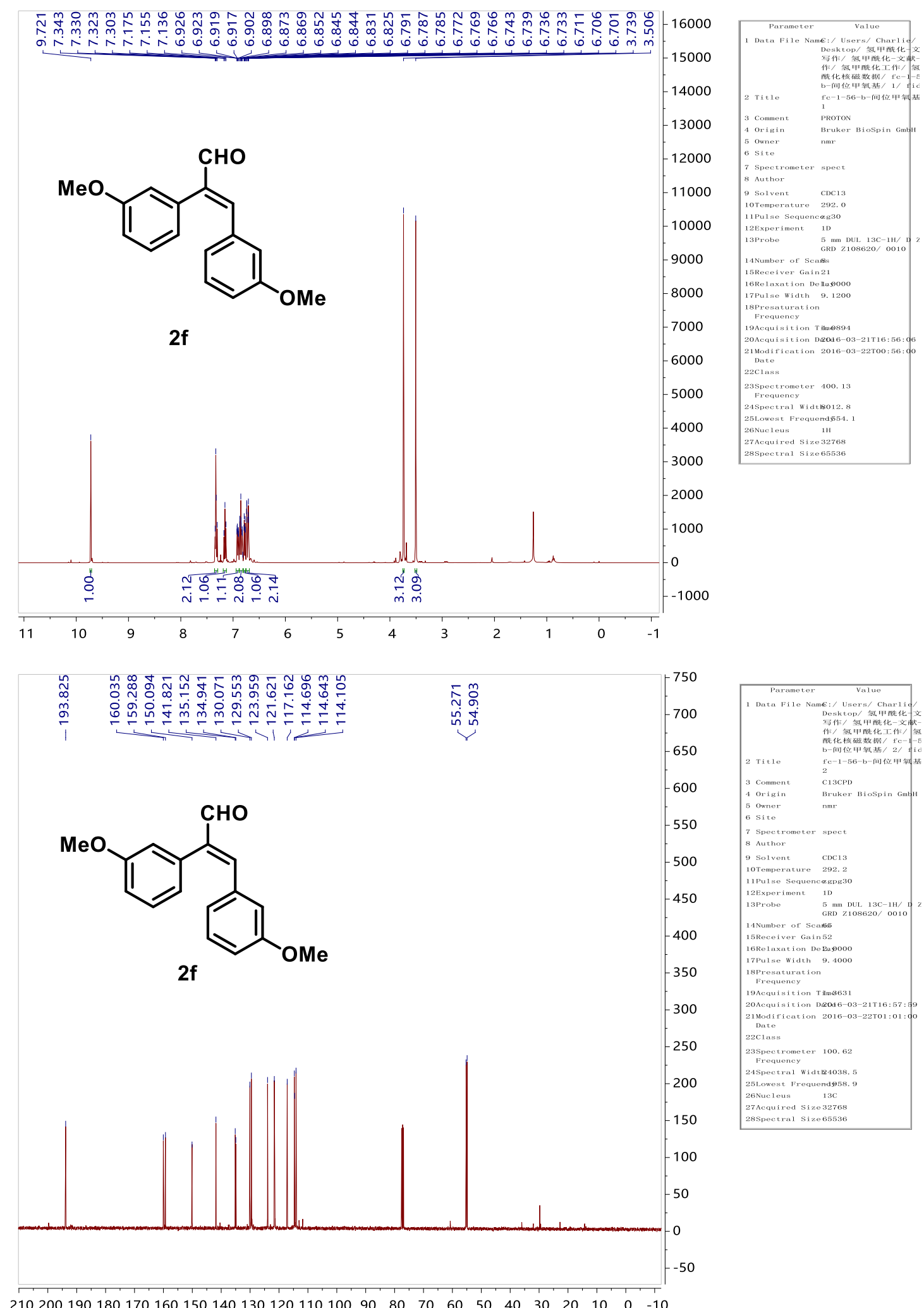


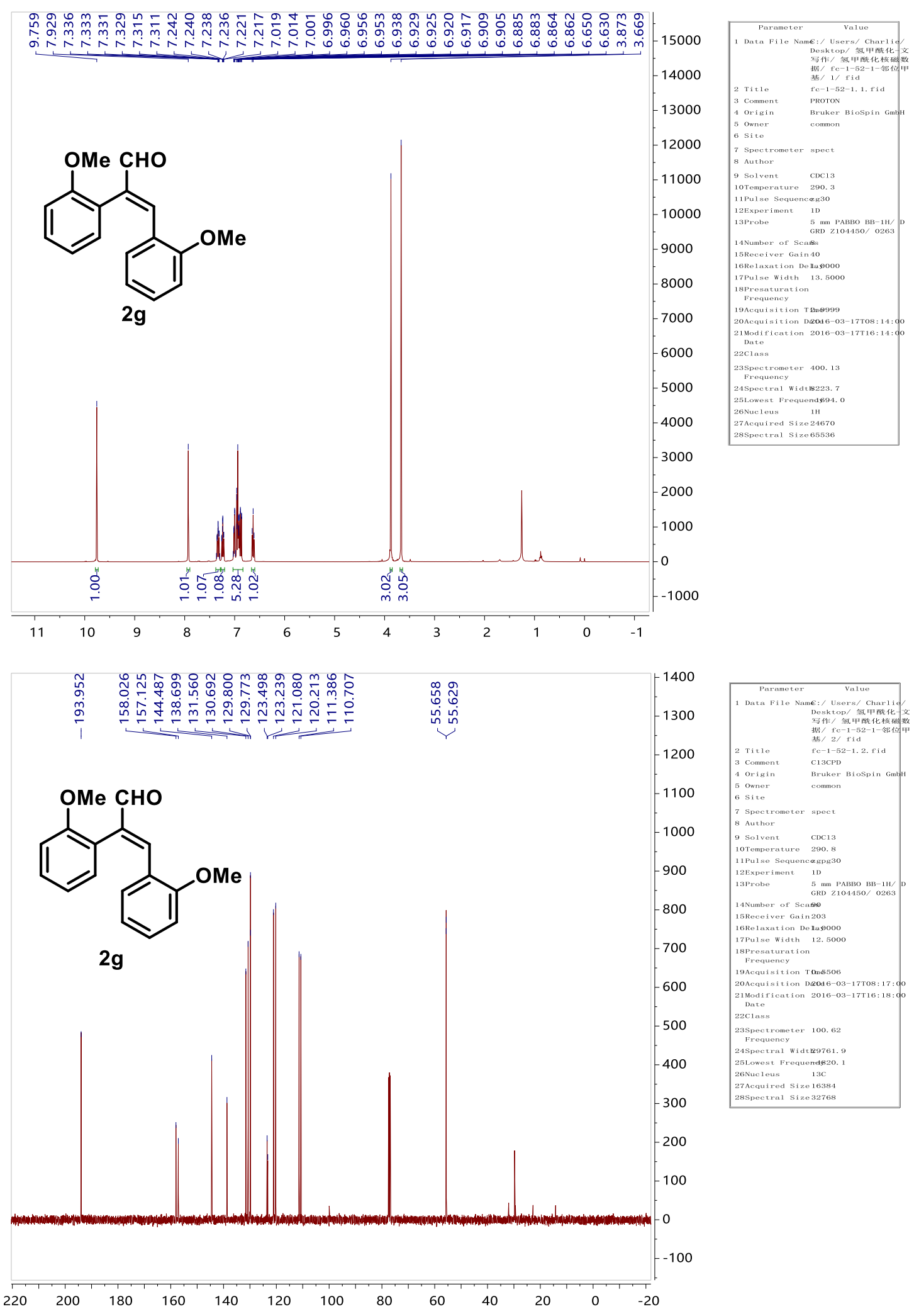



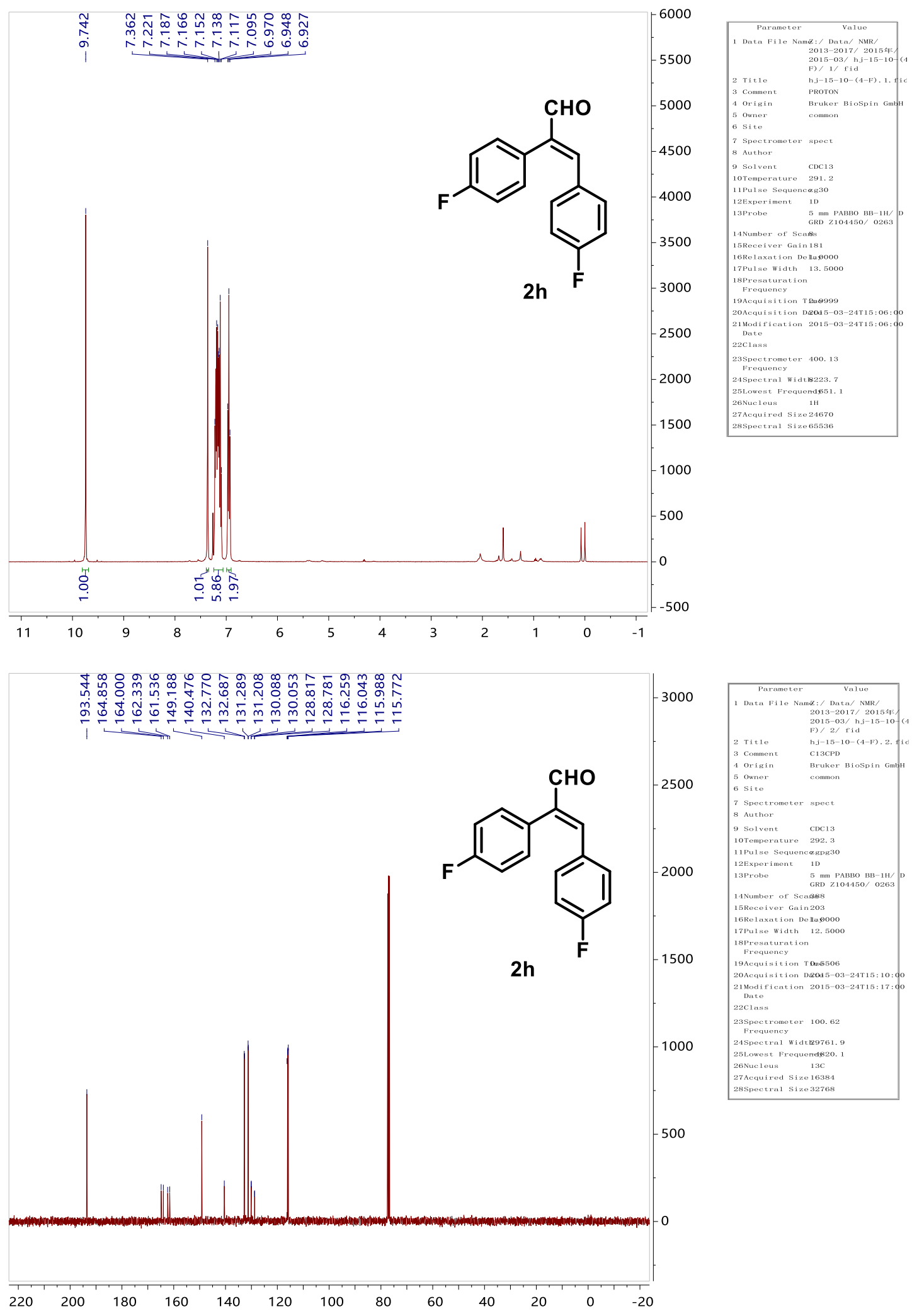


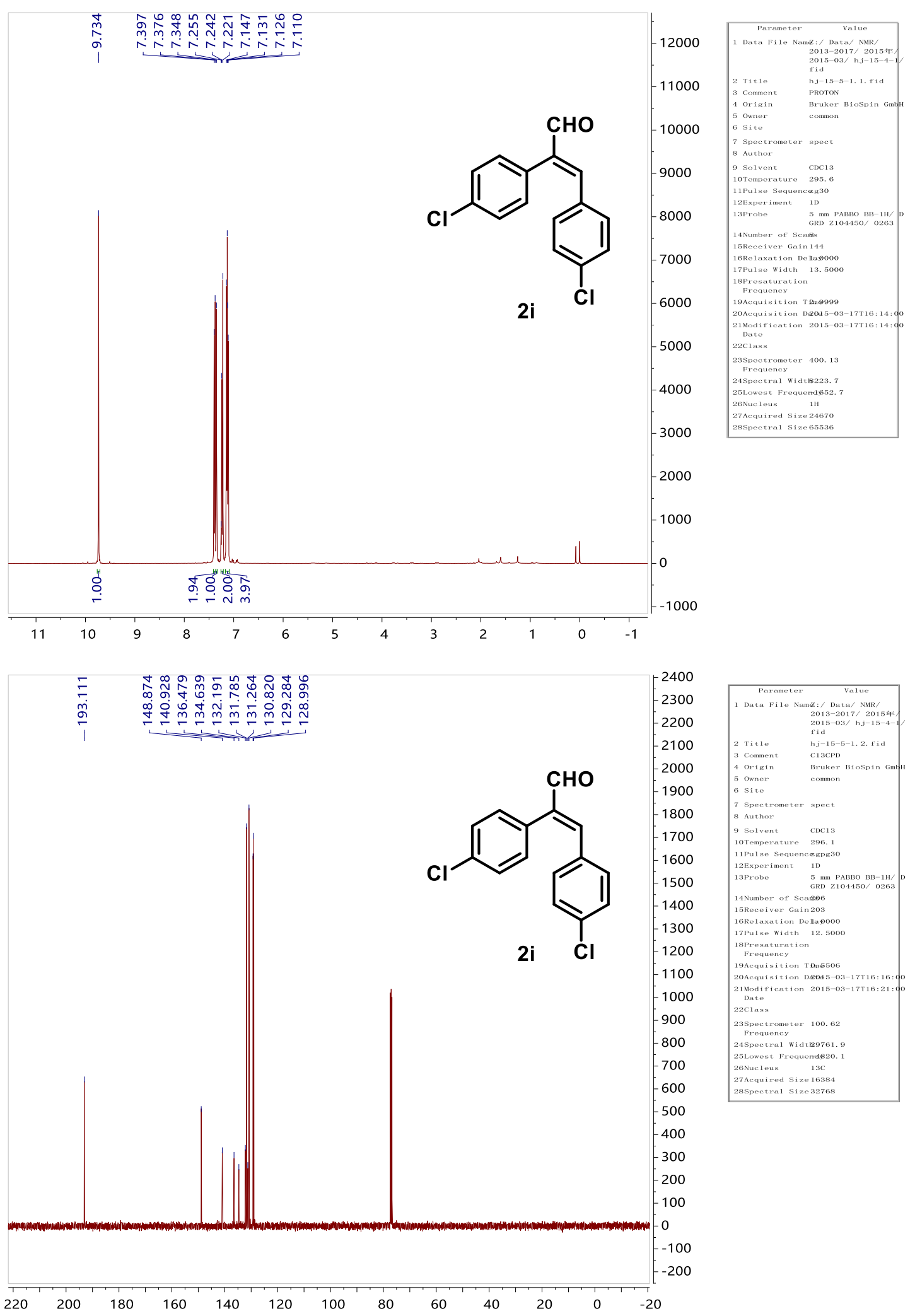



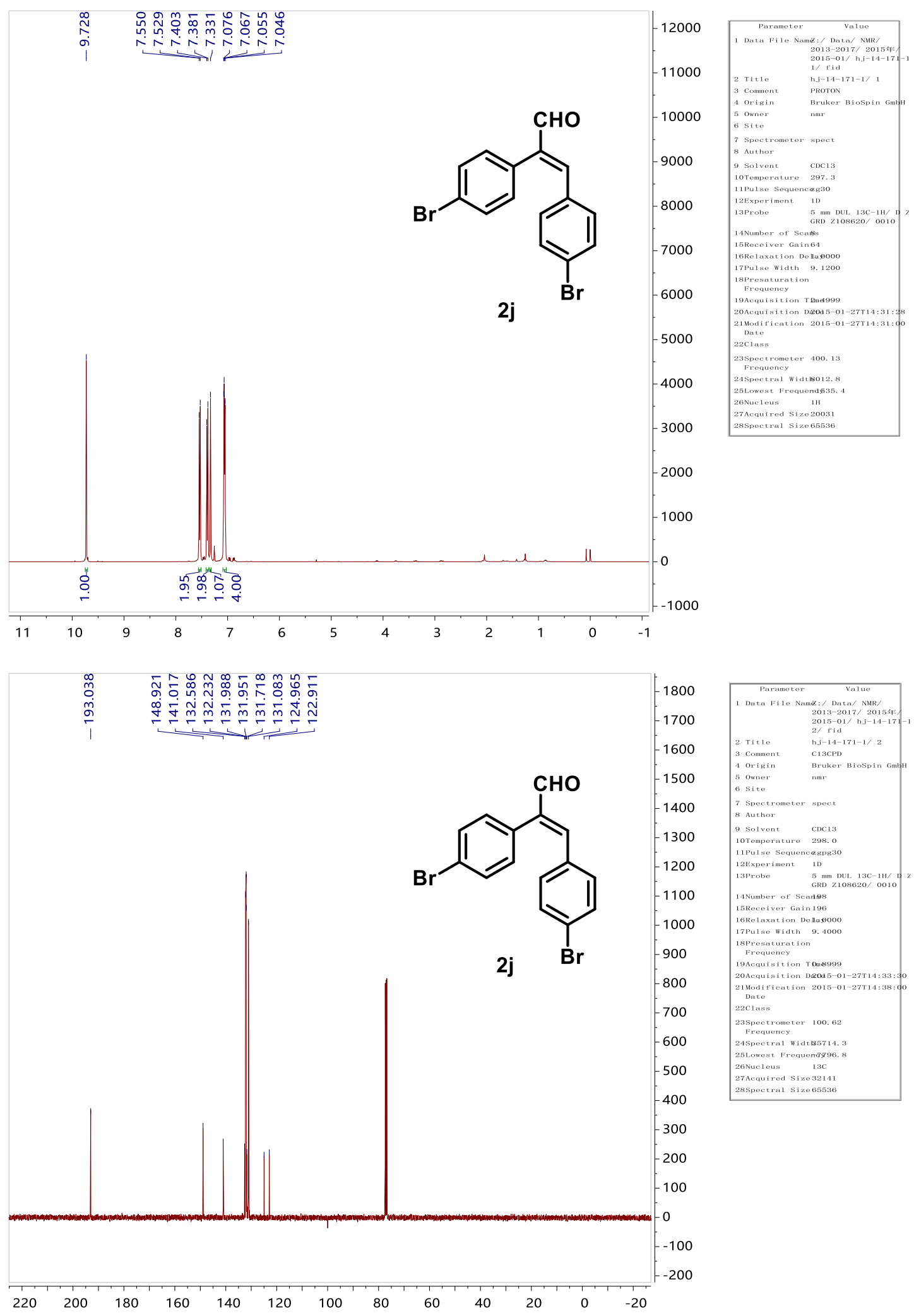

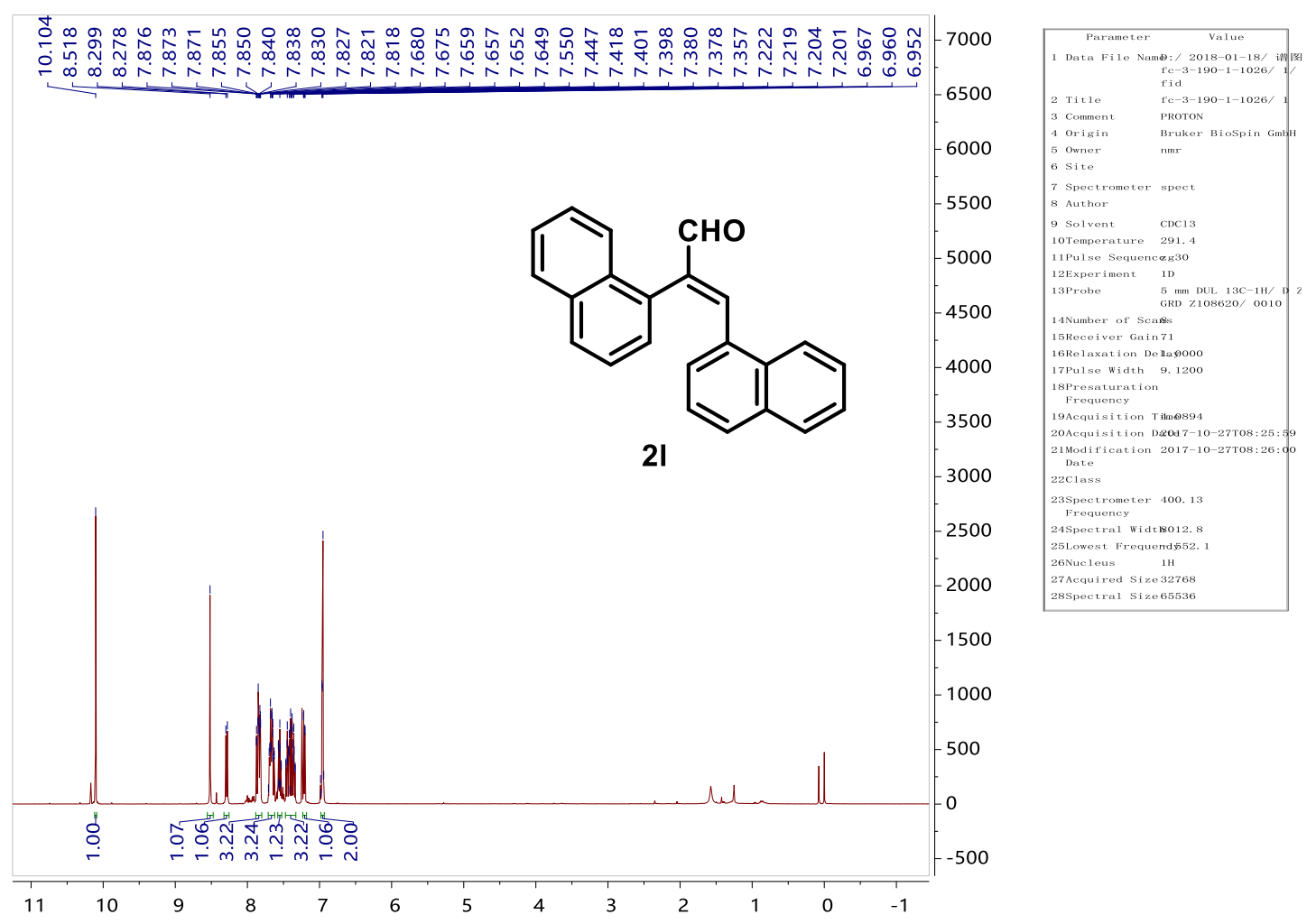

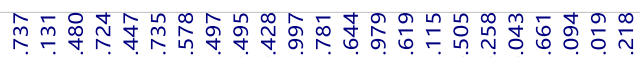

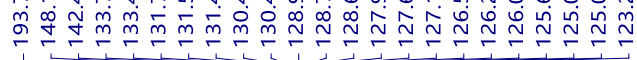
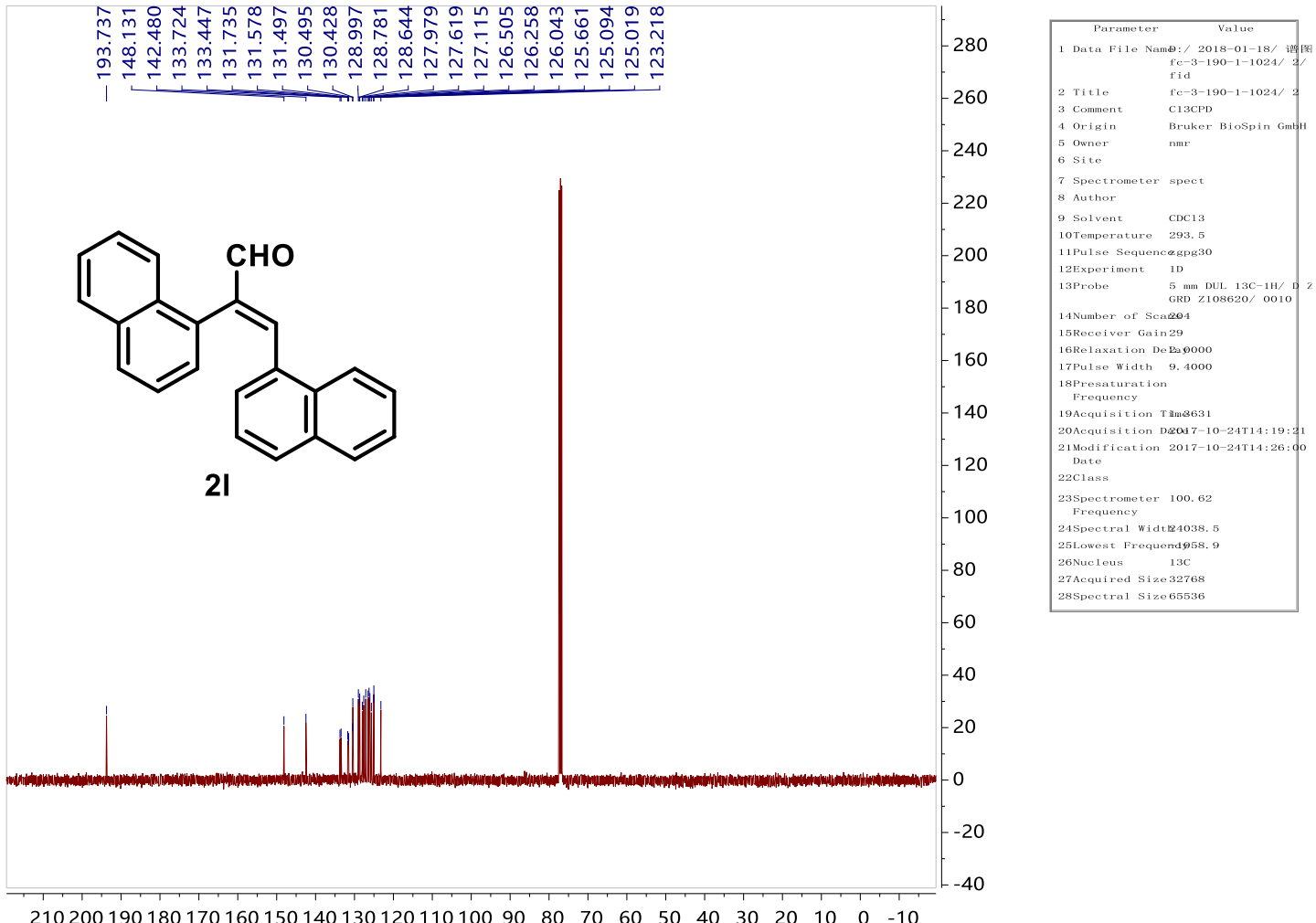


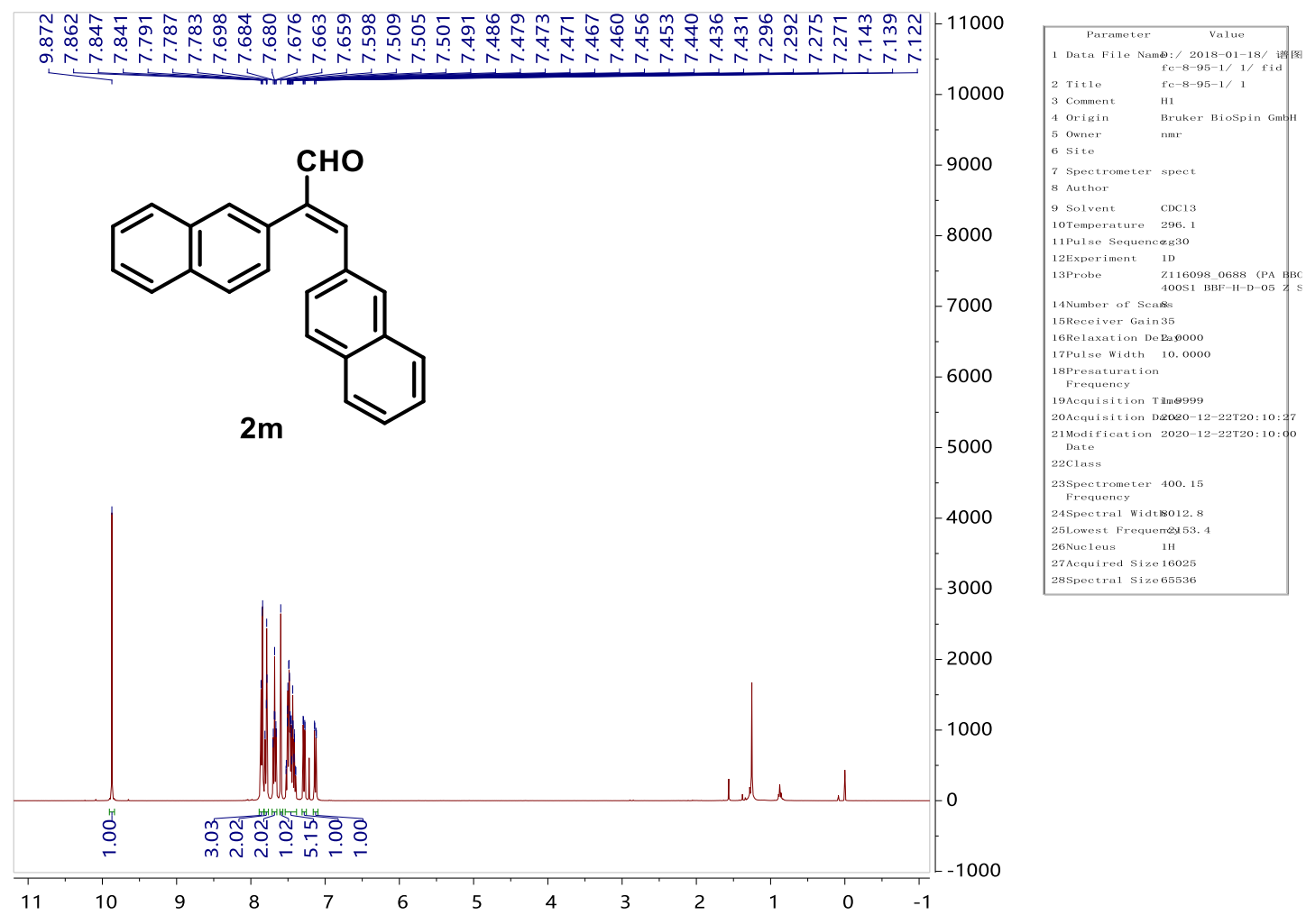

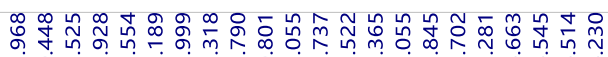

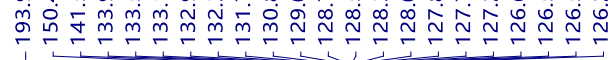<smiles>O=C/C(=C/c1ccc2ccccc2c1)c1ccc2ccccc2c1</smiles>
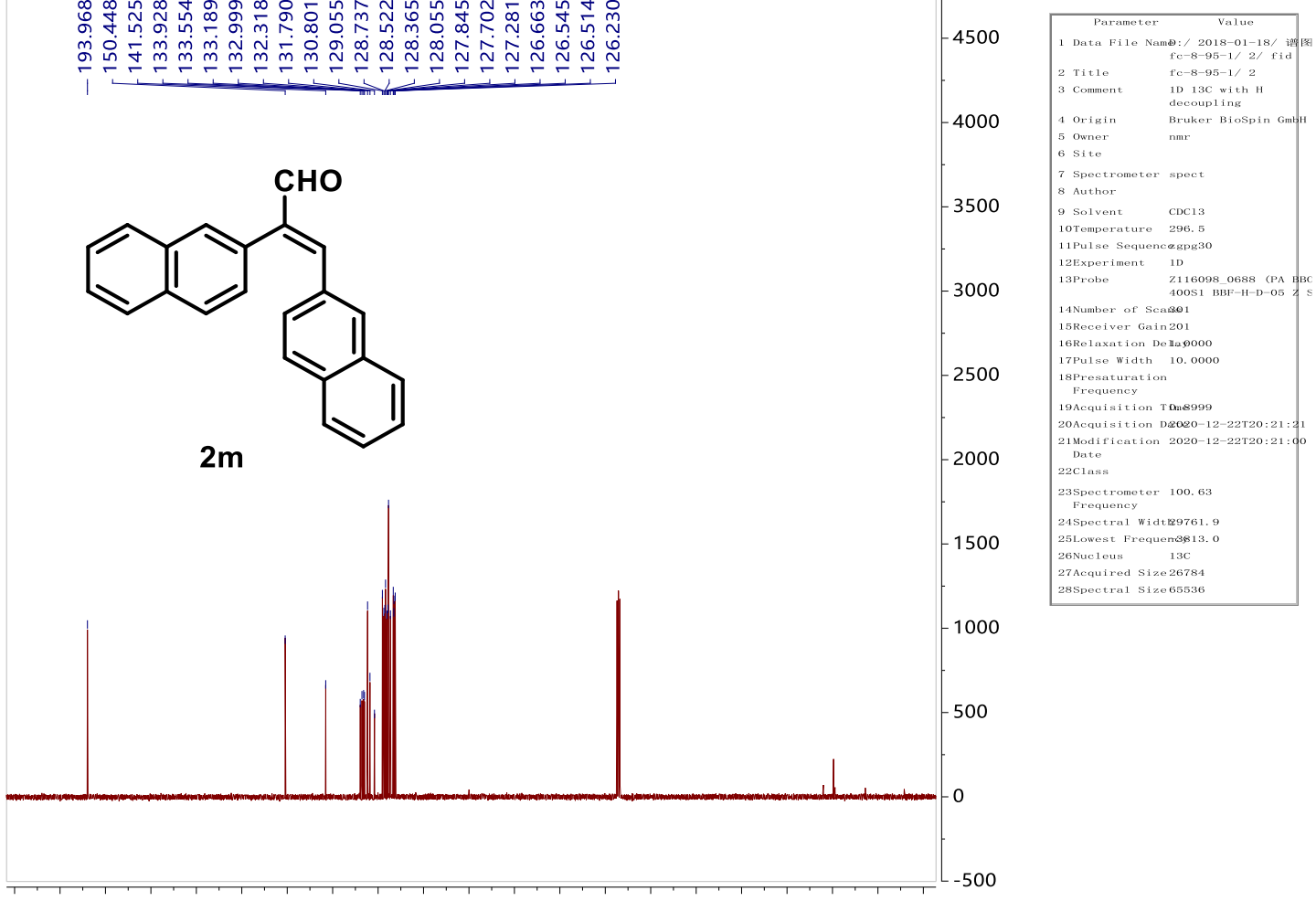

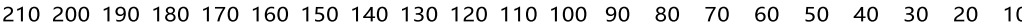




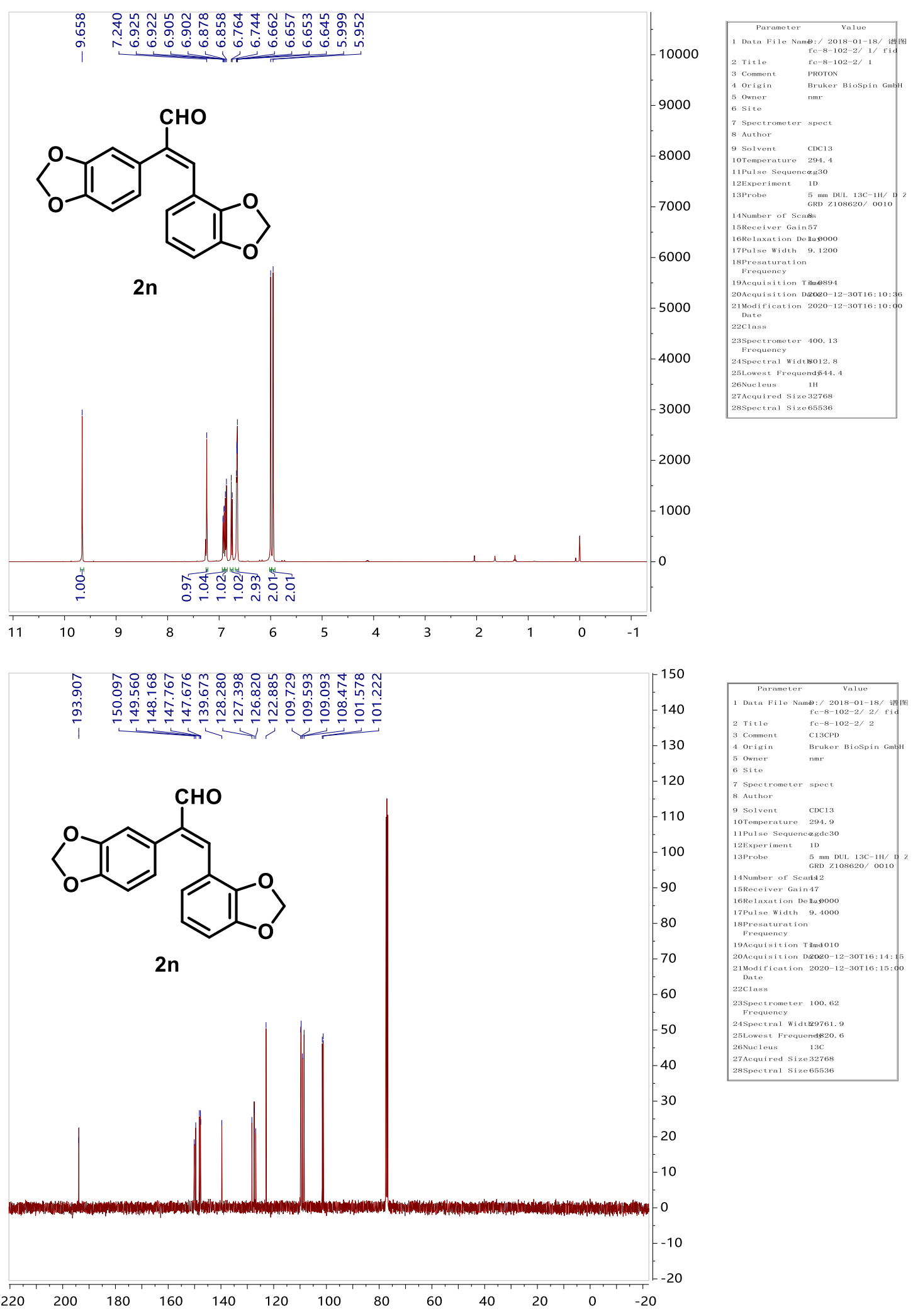



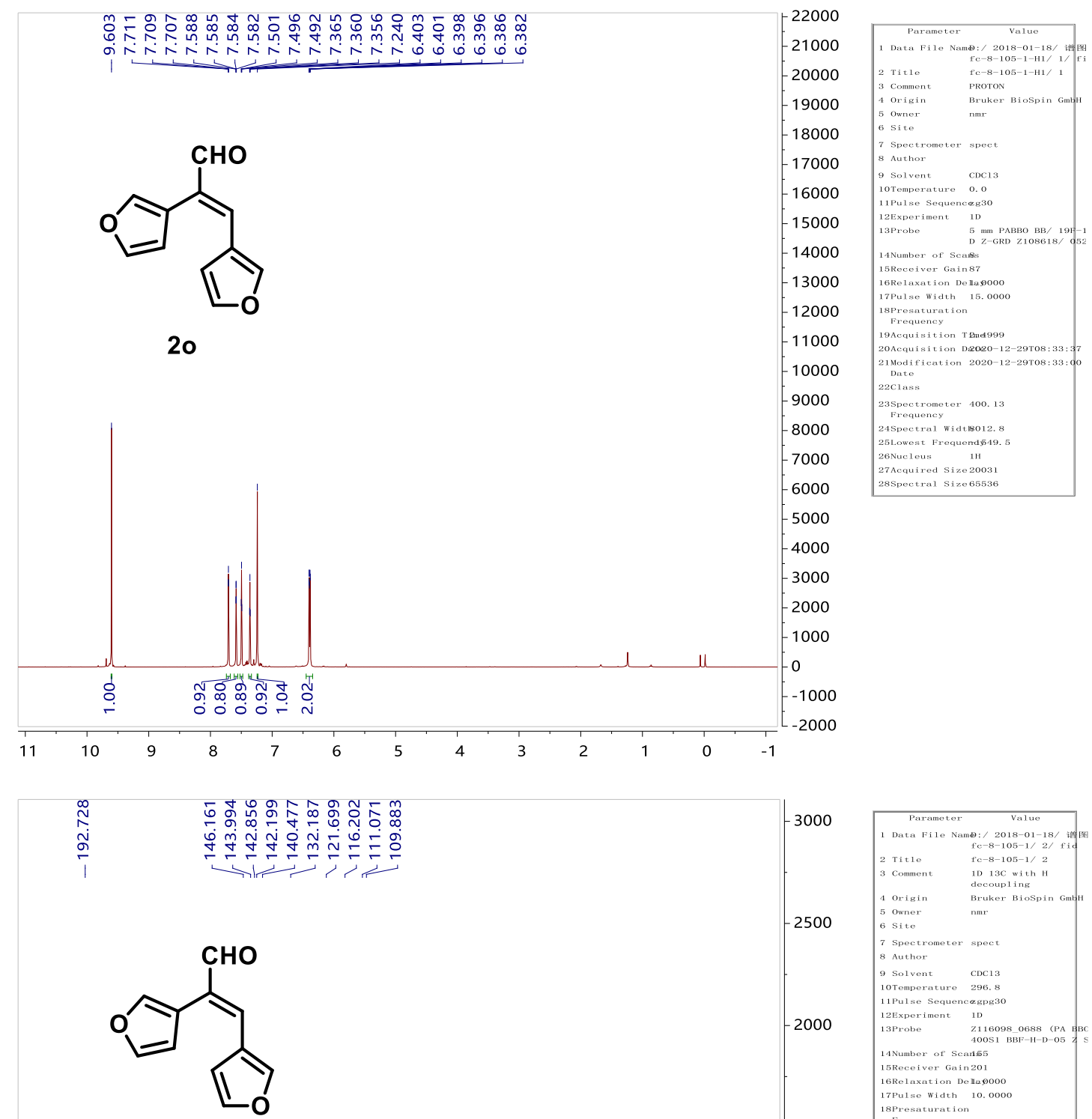

20
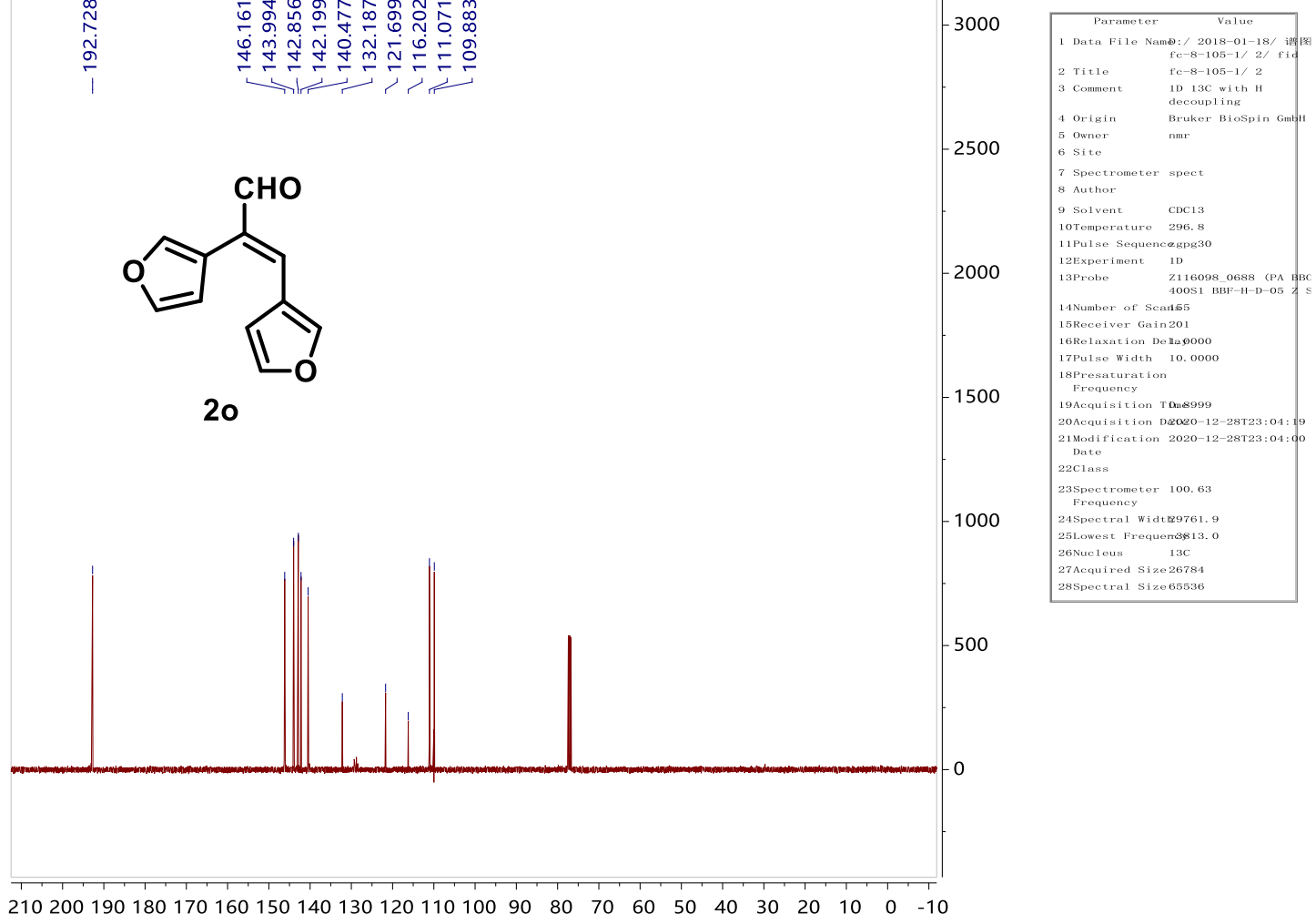

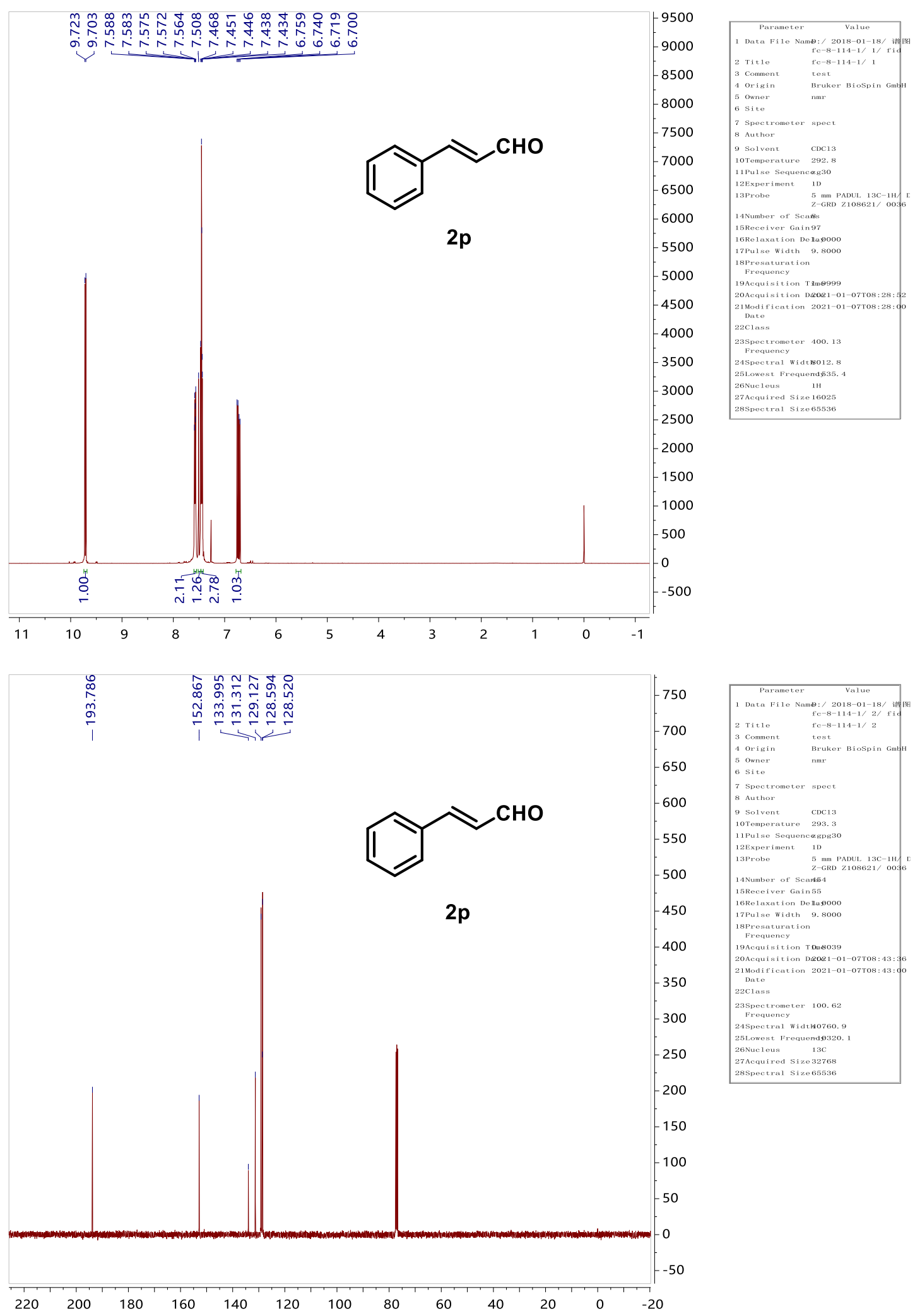

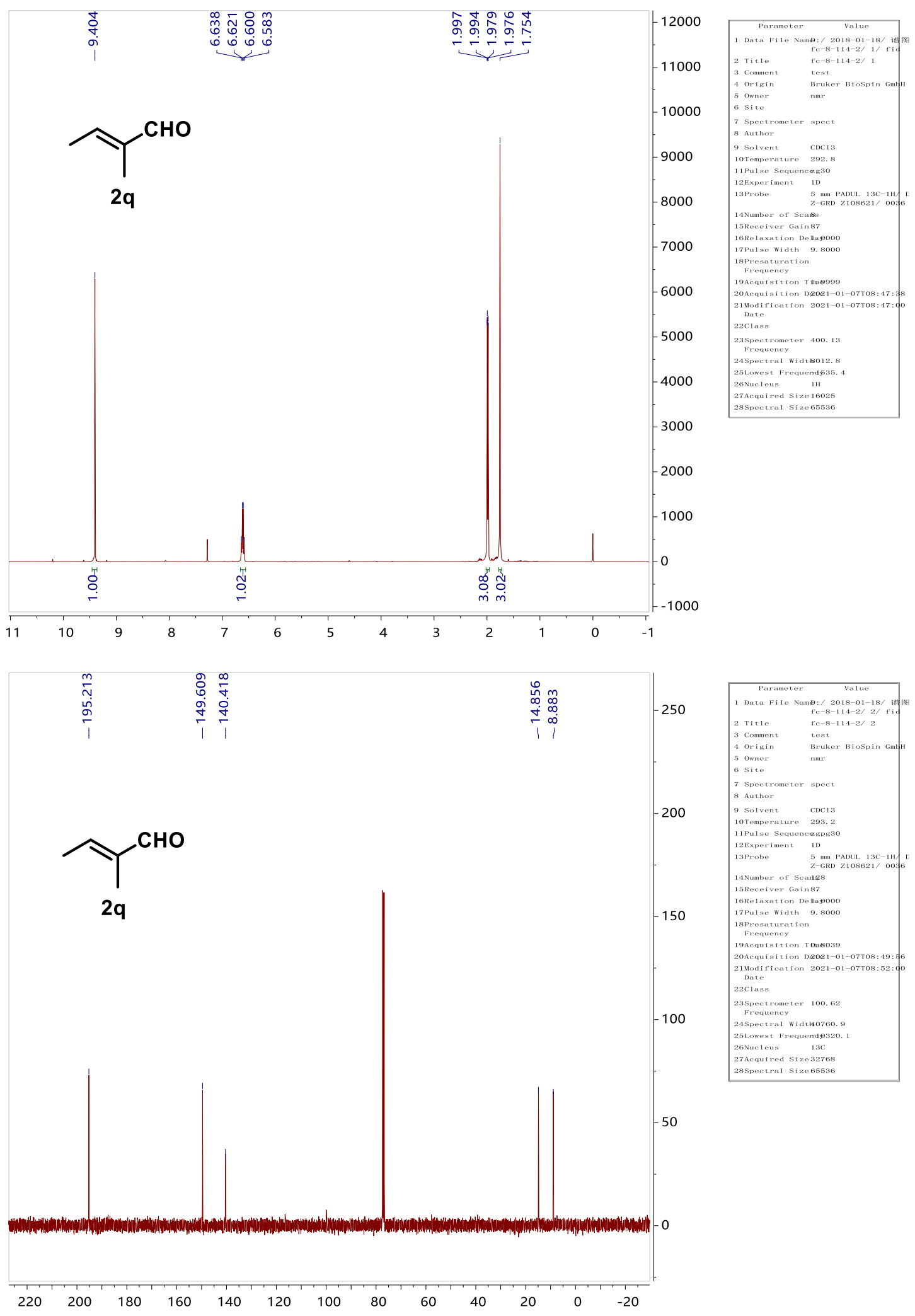


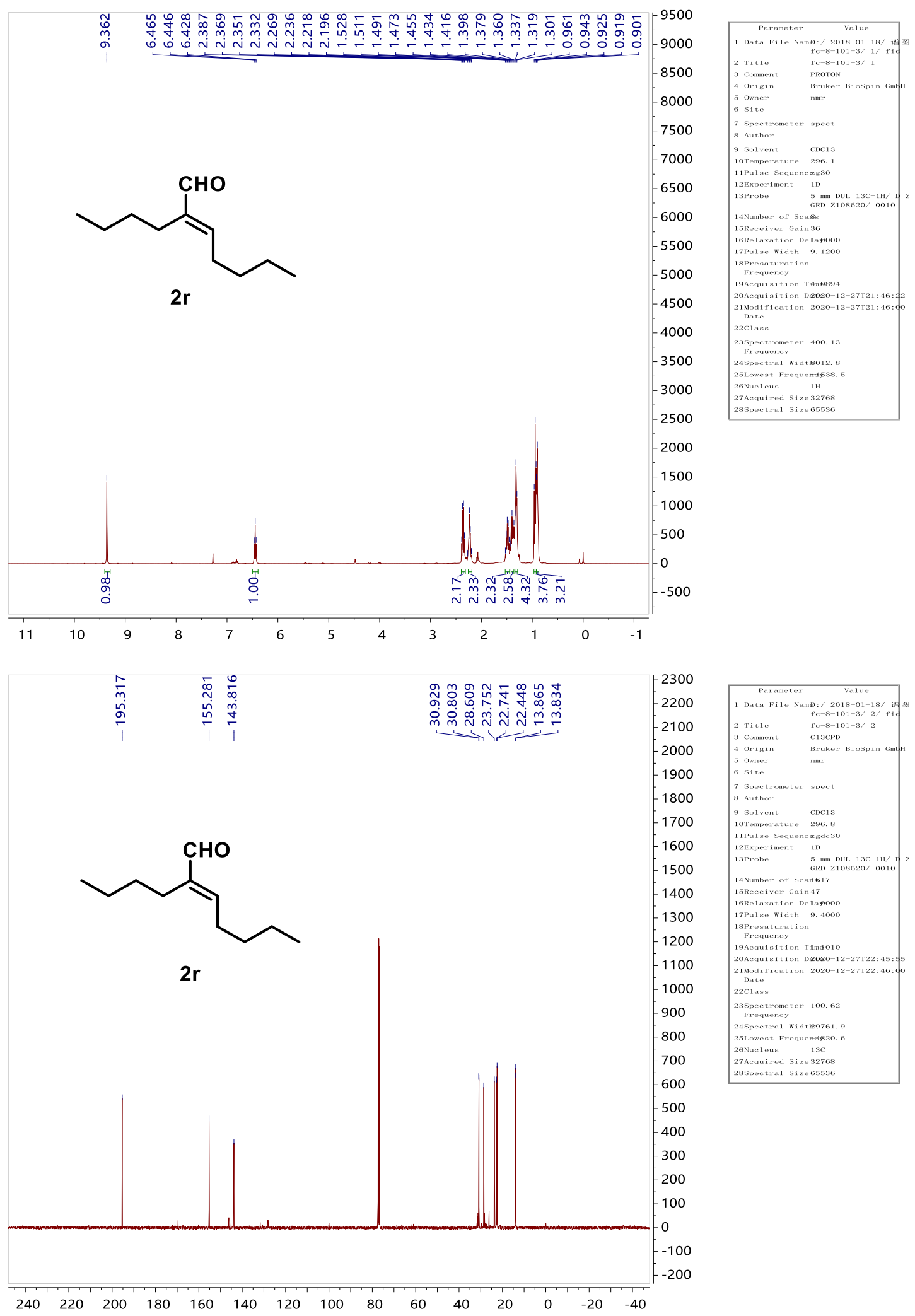




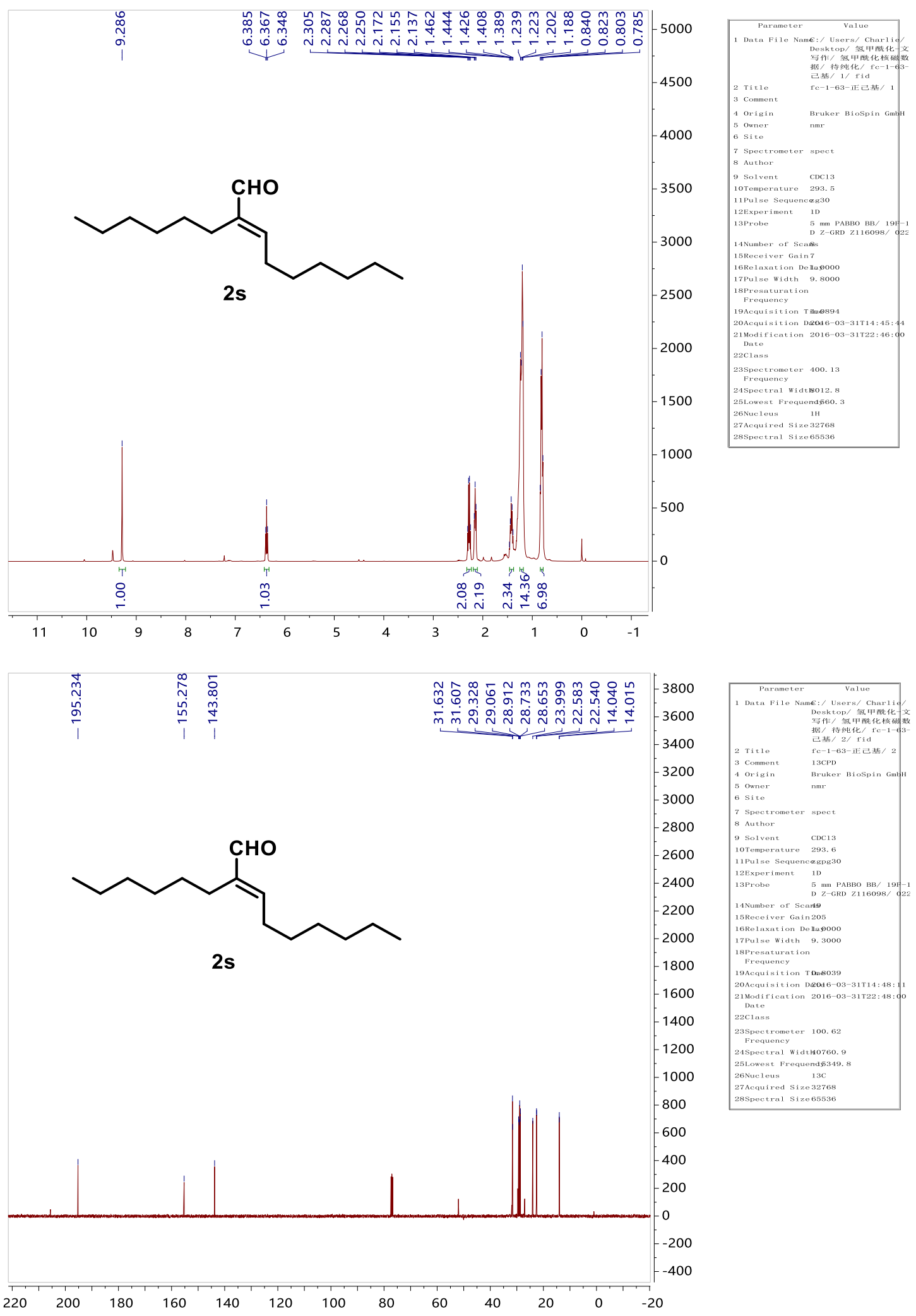



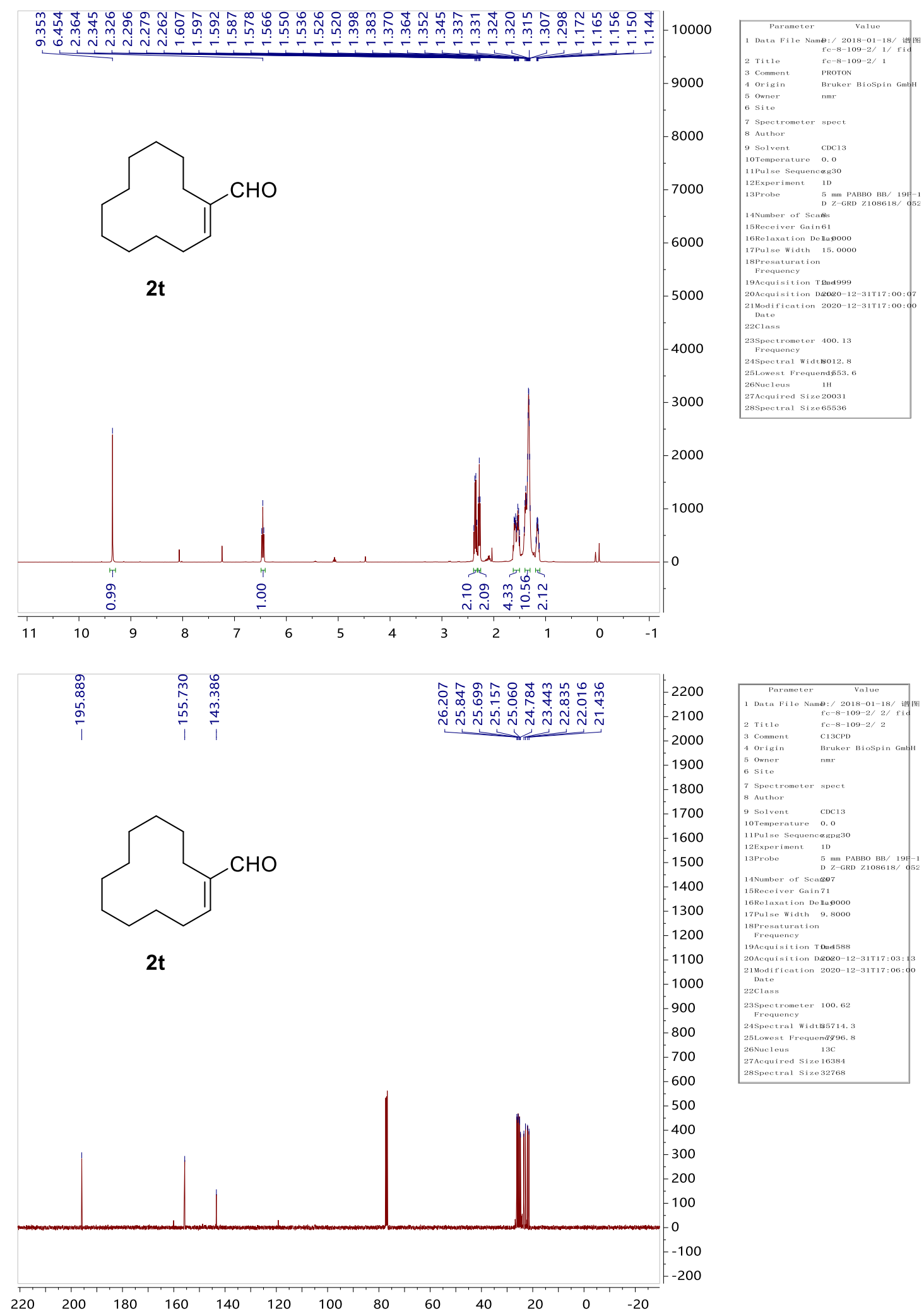

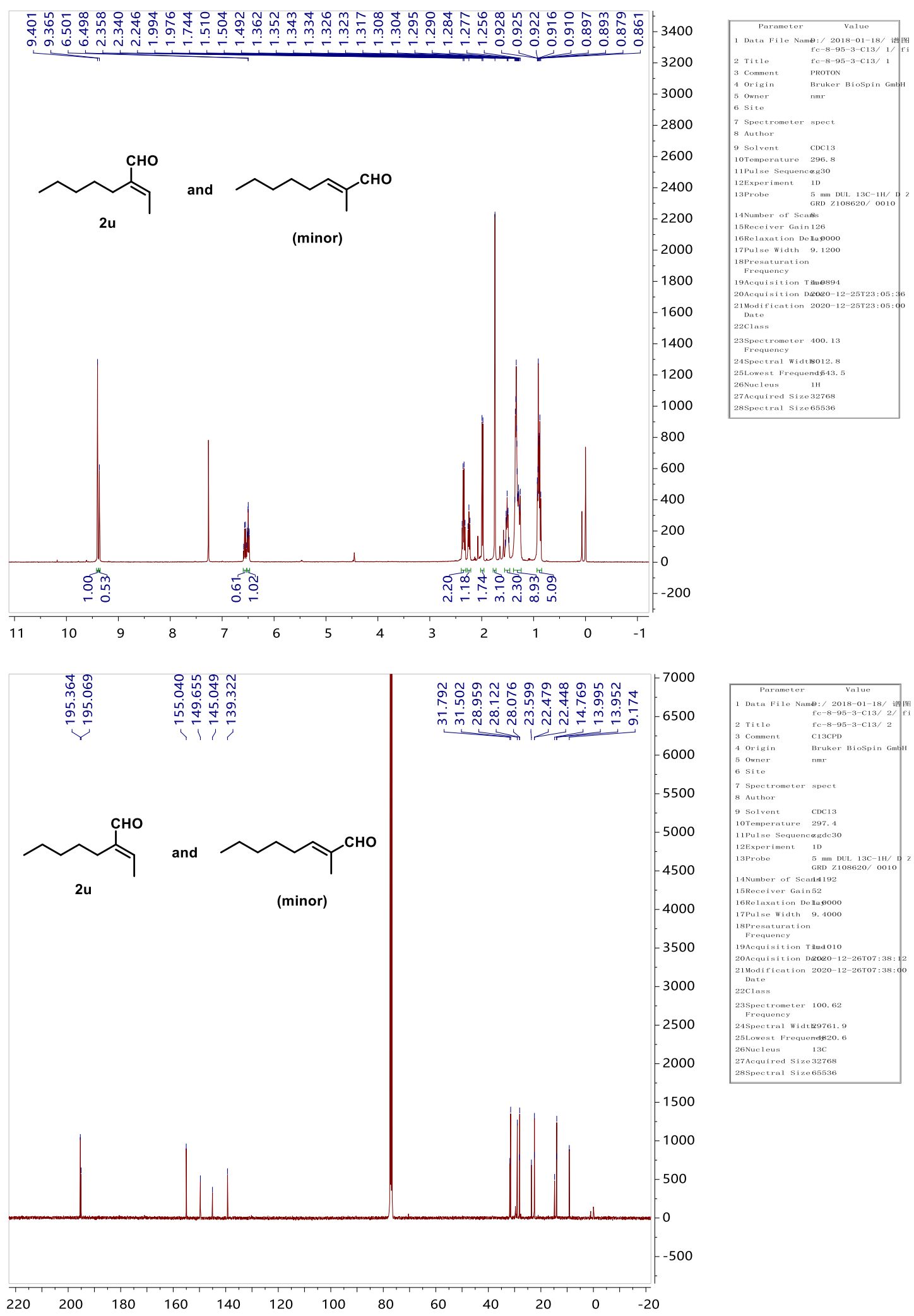

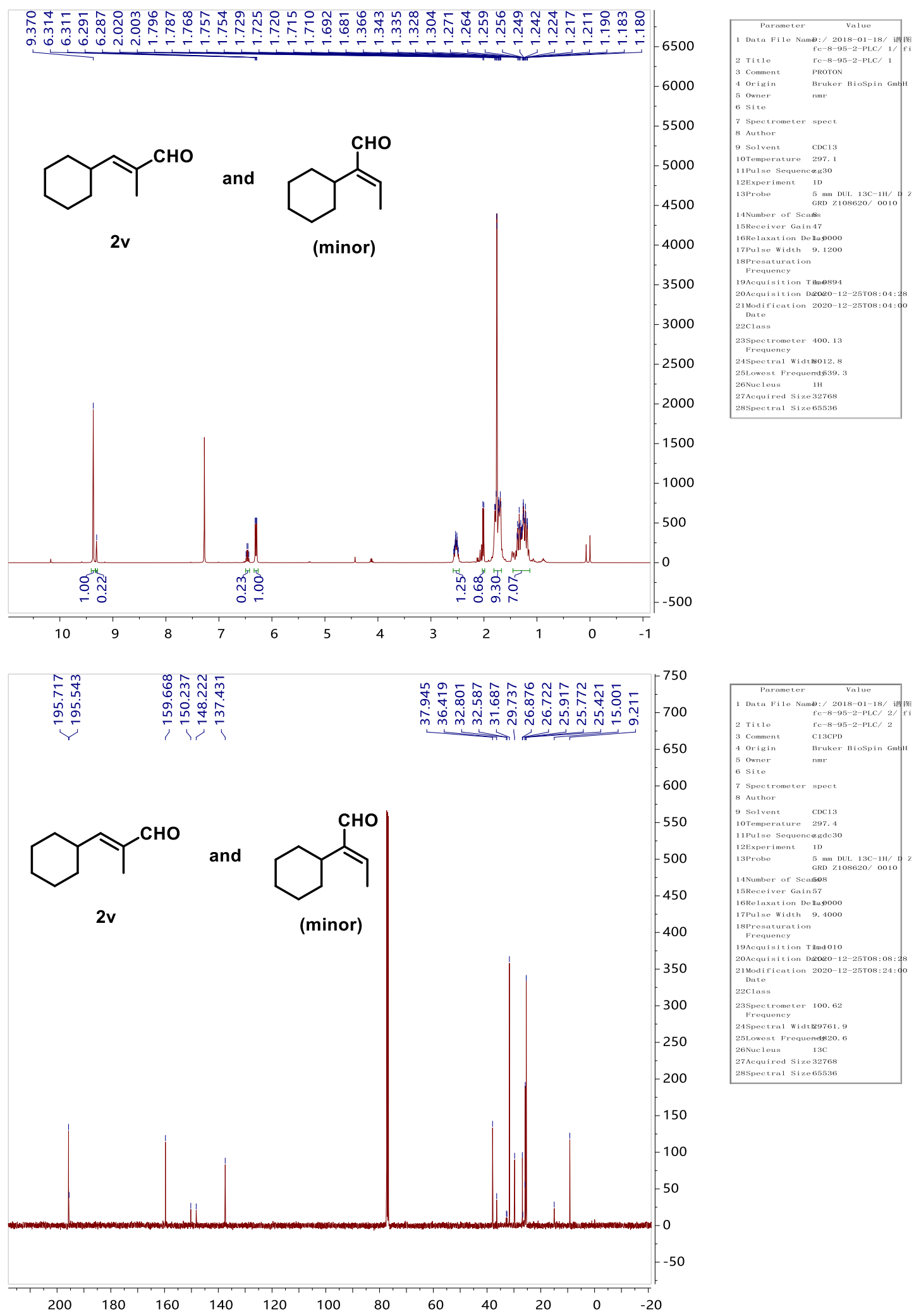


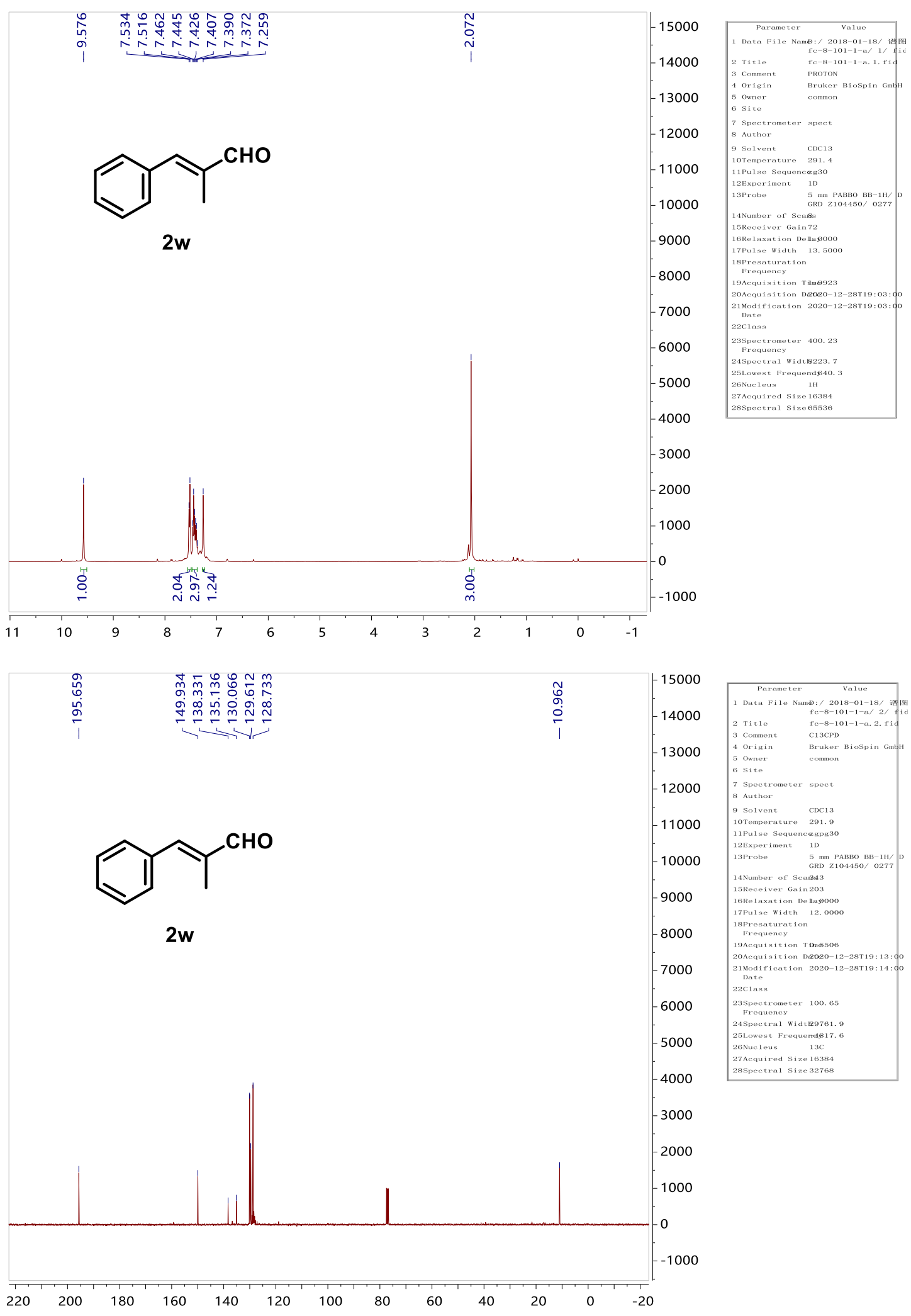




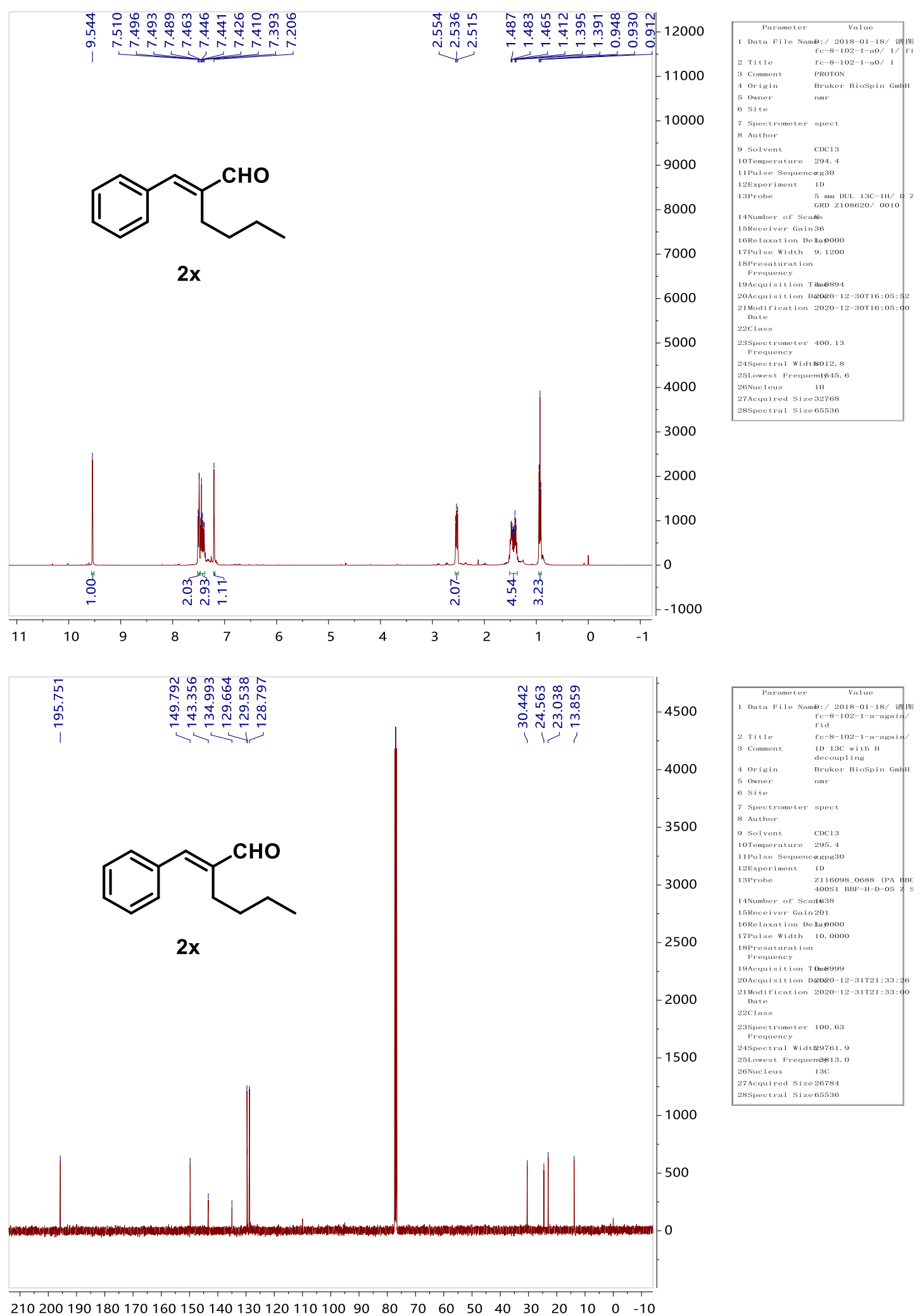



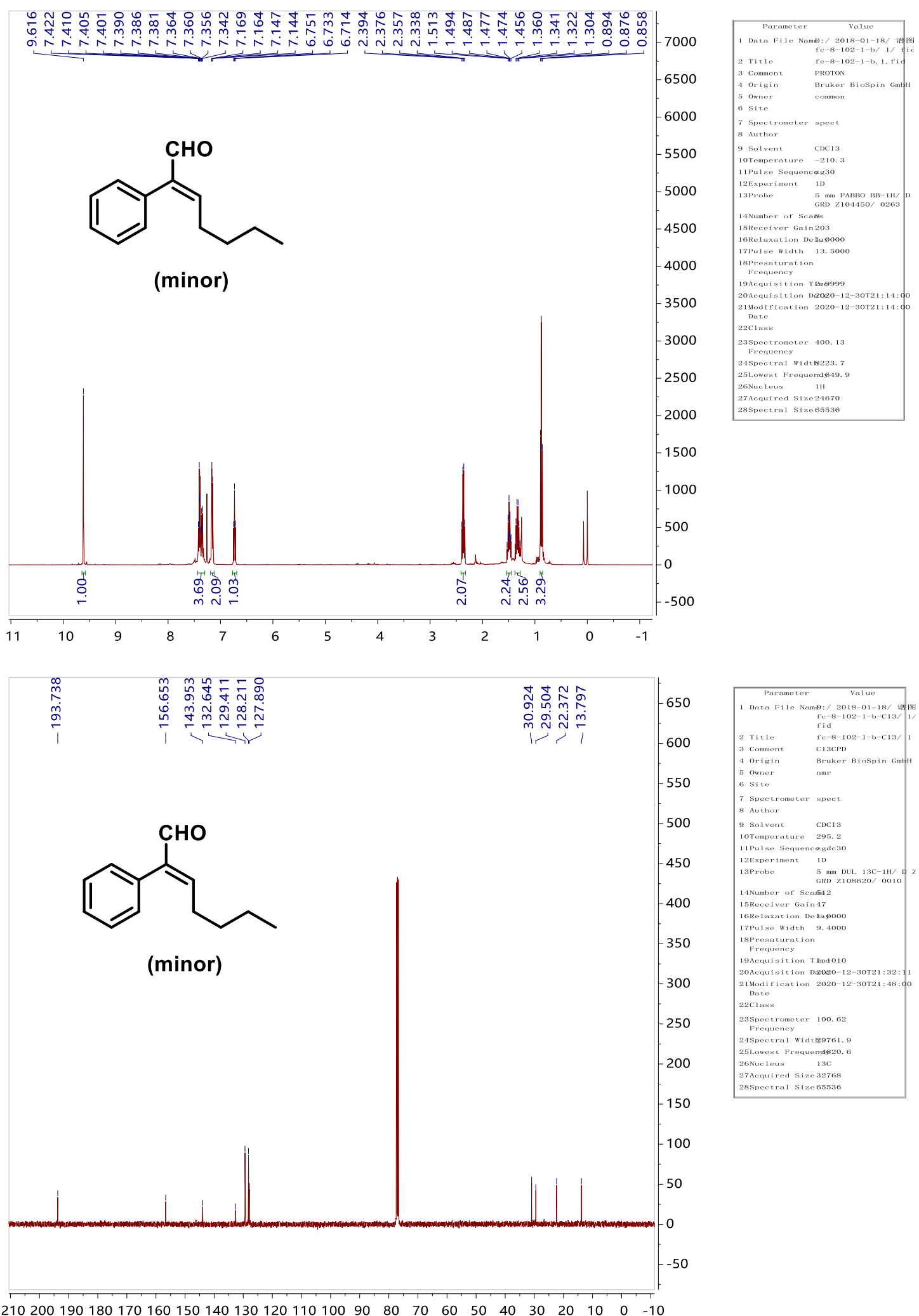

$21020019018017016015014013012011010090 \quad 80 \quad 70 \quad 60 \quad 50 \quad 40 \quad 30 \quad 20 \quad 10 \quad 0 \quad-10$ 


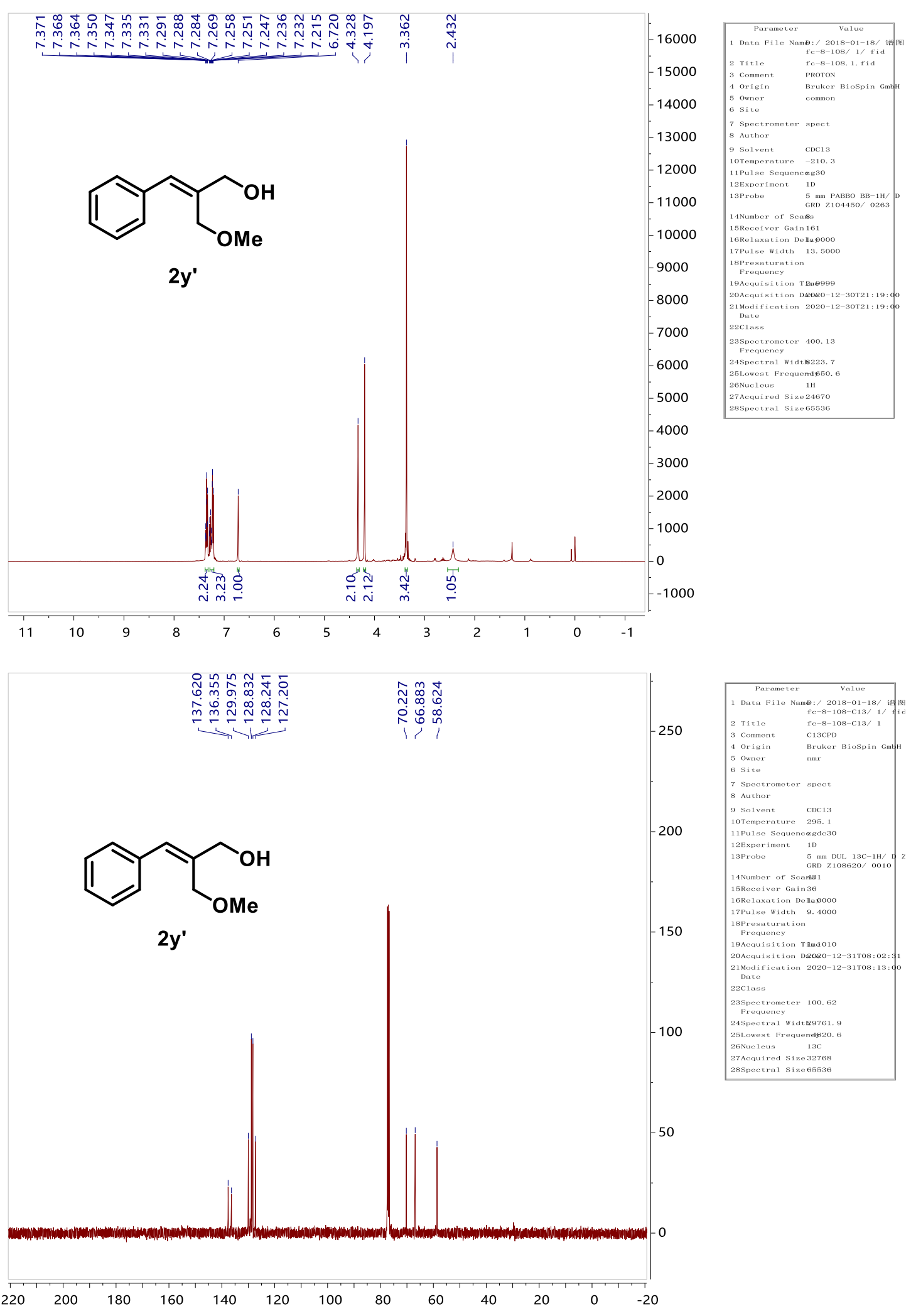




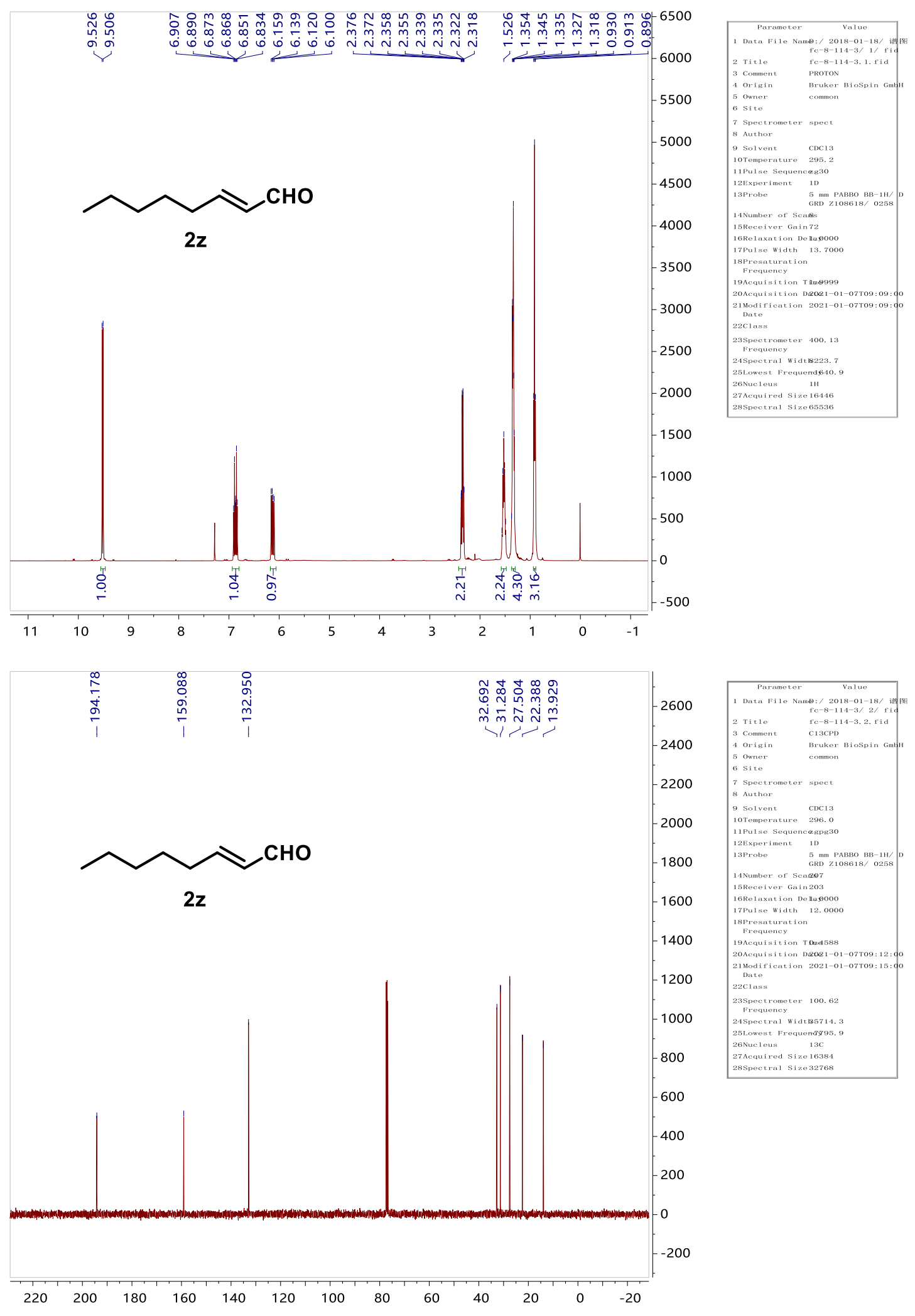




\section{References and Notes}

1. Bonati, F.; Wilkinson, G. Dicarbonyl- $\beta$-diketonato- and related complexes of rhodium(I). J. Chem. Soc. 1964, 3156-3160.

2. Armarego, W. L. F; Chai, C. L. L. in Purification of laboratory chemicals $6^{\text {th }}$ edition. Elsevier: 2009; pp 145.

3. Park, K.; Bae, G.; Moon, J.; Choe, J.; Song, K. H.; Lee, S. Synthesis of symmetrical and unsymmetrical diarylalkynes from propiolic acid using palladium-catalyzed decarboxylative coupling. J. Org. Chem. 2010, 75, 6244-6251.

4. Qiu, S.; Zhang, C.; Qiu, R.; Yin, G.; Huang, J. One-pot domino synthesis of diarylalkynes/1,4-diaryl-1,3- diynes by [9,9-Dimethyl-4,5bis(diphenylphosphino)xanthene]- copper(I) iodine-palladium(II) acetate catalyzed double sonogashira-type reaction. Adv. Synth. Catal. 2018, 360, 313-321.

5. van Vliet, K. M.; Polak, L. H.; Siegler, M. A.; van der Vlugt, J. I.; Guerra, C. F.; de Bruin, B. Efficient copper-catalyzed multicomponent synthesis of $\mathrm{N}$-acyl amidines via acyl nitrenes J. Am. Chem. Soc. 2019, 141, 15240-15249

6. Park, K.; Bae, G.; Park, A.; Kim, Y.; Choe, J.; Song, K. H.; Lee, S. Synthesis of symmetrical diarylalkyne from palladium-catalyzed decarboxylative couplings of propiolic acid and aryl bromides under water Tetrahedron Lett. 2011, 52, 576-580.

7. Li, W.; Yu, S.; Li, J.; Zhao, Y. Nickel-catalyzed allylmethylation of alkynes with allylic alcohols and $\mathrm{AlMe}_{3}$ : facile access to skipped dienes and trienes Angew. Chem. Int. Ed. 2020, 59, 14404-14408.

8. Chen, J.; Chen, C., Chen, J., Wang, G.; Qu, H. Cu-catalyzed intramolecular aryletherification reactions of alkoxyl alkynes with diaryliodonium salts via cleavage of a stable C-O bond Chem Commun. 2015, 51, 1356-1359.

9. Doulcet, J.; Stephenson, G. R. Novel asymmetric formylation of aromatic compounds: enantioselective synthesis of formyl 7,8 dipropyltetrathia[7]helicenes. Chem. E. -J. 2015, 21, 13431-13436.

10. Agabekov, V.; Seiche, W.; Breit, B. Rhodium-catalyzed hydroformylation of alkynes employing a self-assembling ligand system. Chem. Sci. 2013, 4, 2418-2422.

11. Fang, X.; Zhang, M.; Jackstell, R.; Beller, M. Selective palladium - catalyzed hydroformylation of alkynes to $\alpha, \beta$-unsaturated aldehydes Angew. Chem. Int. Ed. 2013, 52, 4645-4649.

12. Zhang, Z.; Wang, Q.; Chen, C.; Han, Z.; Dong, X.-Q.; Zhang, X. Selective rhodiumcatalyzed hydroformylation of alkynes to $\alpha, \beta$-unsaturated aldehydes with a tetraphosphoramidite ligand Org. Lett. 2016, 18, 3290-3293.

13. Tan, G.; Wu, Y.; Shi, Y.; You, J. Syngas-free highly regioselective rhodium catalyzed transfer hydroformylation of alkynes to $\alpha, \beta$-unsaturated aldehydes Angew. Chem. Int. Ed. 2019, 58, 7440-7444.

14. Barbot, F.; Miginiac, P. Preparation of 3-alkenals and 3-alkynals by hydrolysis of the corresponding acetals $\mathbf{1 9 8 3}, 14,651-654$.

15. Fang, Bruch, A.; Breit, B. Tandem-directed regioselective hydroformylation/ $\beta$ elimination: a practical method for the synthesis of enals Synthesis. 2008, 14, 2169 2176. 
16. Guo, W.; Kuniyil, R.; Gómez, J. E.; Maseras, F.; Kleij, A. W. A domino process toward functionally dense quaternary carbons through Pd-catalyzed decarboxylative $\mathrm{C}\left(\mathrm{sp}^{3}\right)-\mathrm{C}\left(\mathrm{sp}^{3}\right)$ bond formation. J. Am. Chem. Soc. 2018, 141, 39813987.

17. Brenna, E.; Gatti, F. G.; Monti, D.; Parmeggiani, F.; Sacchetti, A. Cascade coupling of ene reductases with alcohol dehydrogenases: enantioselective reduction of prochiral unsaturated aldehydes ChemCatChem 2012, 4, 653-659.

18. van Loon, W. A. M.; Linssen, J. P. H.; Beolrijk, A. E. M.; Burgering, M. J. M.; Voragen, A. G. J. Real-time flavor release from french fries using atmospheric pressure chemical ionization-mass spectrometry. J. Agric. Food Chem. 2005, 53, 6438-6442. 